\title{
Entrelacs
}

Cinéma et audiovisuel

Hors-série $n^{\circ} 4$ | 2016

Paysages en séries

\section{Entre panorama et ornement : le paysage antique dans les séries (2000-2015)}

Entre panorama et ornement: le paysage antique dans les séries (2000-2015)

\section{Laury-Nuria André}

\section{(2) OpenEdition}

Journals

\section{Édition électronique}

URL : http://journals.openedition.org/entrelacs/2141

DOI : 10.4000/entrelacs. 2141

ISBN : $2261-5482$

ISSN : 2261-5482

Éditeur

Éditions Téraèdre

Édition imprimée

Date de publication : 1 novembre 2016

ISSN : 1266-7188

\section{Référence électronique}

Laury-Nuria André, «Entre panorama et ornement : le paysage antique dans les séries (2000-2015)»,

Entrelacs [En ligne], Hors-série $n^{\circ} 4 \mid$ 2016, mis en ligne le 06 février 2018, consulté le 20 avril 2019.

URL : http://journals.openedition.org/entrelacs/2141; DOI : 10.4000/entrelacs.2141

Ce document a été généré automatiquement le 20 avril 2019.

Tous droits réservés 


\title{
Entre panorama et ornement : le paysage antique dans les séries (2000-2015)
}

Entre panorama et ornement : le paysage antique dans les séries (2000-2015)

\author{
Laury-Nuria André
}

1 Il existe, dans les nombreux champs du savoir académique et dans les nobles disciplines humanistes en péril, des idées tenaces - fort belles et dont la teneur historique n'est pas fausse, seulement vraie partiellement - à partir desquelles toute une discipline et tout un regard culturel se façonnent et façonnent en retour notre rapport actuel à l'Art, à la théorie et à la pratique artistiques. L'une des plus tenaces est sans doute celle qui veut que le paysage ne naisse - conceptuellement et artistiquement - qu'aux prémices de la Renaissance européenne ${ }^{1}$. Quant à savoir si c'est la Renaissance picturale du Nord qui a, la première, inauguré le genre paysager ou bien si c'est celle des écoles italiennes au Sud, nous laissons-là ces querelles de chapelle aux spécialistes ${ }^{2}$. Il est d'autres courants de pensées actuels qui ont produit une série de travaux entre les années 1990 et 2010, notamment parmi les spécialistes des Sciences de l'Antiquité afin de démontrer, par l'Histoire, par l'Histoire de l'Art, par la Littérature et par l'Archéologie, l'existence du paysage dans les sociétés anciennes ${ }^{3}$. Appartenant à ces courants de pensée, nous considérons que la question est réglée : les Anciens, Grecs et Romains, vivaient avec l'idée, avec les représentations artistiques et littéraires, avec les pratiques, du paysage. Ses formes et ses fonctions différaient certes des nôtres, mais la sensibilité paysagère et la place que le paysage a pu occuper au quotidien, dans la société antique ont été prouvé par toutes sortes de documents: description et œuvres littéraires, textes géographiques, cartes, récits nautiques et périplographiques, textes théoriques et rhétoriques, papyrologie, sculptures, peintures, céramiques etc ${ }^{4}$.

2 Ainsi, de notre point de vue, parler de paysage antique ne constitue ni un anachronisme ni une erreur épistémologique, mais c'est un discours que l'on peut tenir scientifiquement et culturellement. Reste à savoir quel est véritablement le paysage que l'on observe lorsque, spectateur d'une série télévisuelle traitant du monde antique, nous 
souhaitons en apprécier la teneur : relève-t-il d'une reconstitution qui s'appuie sur les documents iconographiques antiques que l'archéologie a pu nous livrer - ceux-là même qui nous donnent à voir quelle morphologie et quelle dimension artistique et culturelle le paysage avait dans la société antique - ou bien s'agit-il d'une représentation plus libre de la part des scénaristes et de la production en général, d'une représentation et d'une réinterprétation qui témoigneraient alors du processus complexe de réception de la matière antique ${ }^{5}$. C'est plus en termes de réception que nous tenterons de voir comment, le paysage des séries télévisuelles à thème antique, du fait des singularités génériques propre au fonctionnement d'une série, permet en retour de questionner la dimension théorique de la définition esthétique du paysage grâce au détour par l'Antique. C'est une approche que nous développons depuis plusieurs années dans nos travaux qui interrogent l'impact de la spécificité des médiums contemporains sur les processus de réception et de transmission de la matière antique ${ }^{6}$.

Un des terrains d'enquête singulier pour mesurer ce travail de réception-création de la matière antique au travers de la question du paysage est l'univers vidéoludique. La singularité du médium, les codes et les enjeux des jeux vidéos sont propres à notre culture actuelle et nous livre des informations précieuses sur l'analyse des images et sur les questions d'Esthétique qui leur sont relatives. Or, il a récemment été montré comment, par le paysage, une ligne de fracture esthétique se met en place dans les péplums des années 2000-2010, sous l'influence des techniques 3D tirant le cinéma hollywoodien du côté de l'esthétique des jeux vidéo8. Plus de ruines mais des panoramas grandioses et des anastyloses virtuelles'. Dans ce paysage, la ligne d'horizon est repoussée, les lignes de fuite, et les perspectives sont arrondies, la courbe dilate l'espace ${ }^{10}$ : l'Antiquité est matière à un rêve paysager dont l'incommensurabilité est gage d'abolition des frontières chronologiques et culturelles qui séparent notre société de cette dernière. L'esthétique vidéoludique et cinématographique des péplums en témoigne.

4 Le passage au petit écran et à la sérialité, répète-t-il ce paradigme paysager de l'Antique ou bien en émerge-t-il un propre à la logique du " genre » sériel ? Rome $(2005, \mathrm{HBO})^{11}$, par sa mise en scène méticuleuse des realia antiques - décor studio, grandeur nature ou tournage en plein air - s'impose à notre enquête. En la comparant à deux autres séries à thème antique, à Atlantis (2013, BBC One $)^{12}$ et à Odysseus, (2013, ARTE) $)^{13}$, Rome permet de dégager une gradation, par rapport au paysage des péplums contemporains, dans l'utilisation du paysage naturel et du paysage virtuel: le schème du panoramique, dominant pourtant la représentation paysagère en Occident moderne $\mathrm{e}^{14}$, se trouve alors congédié dans Atlantis et Odysseus selon deux modalités opposées de métissage des types de paysage.

5 Confronté à l'univers intégralement recomposé dans la série Rome, non pas virtuellement mais artistiquement et artisanalement, le paysage naturel réel ne court-il pas le risque de jouer le rôle d'incongruité visuelle au même titre (mais pour des raisons inverses) que le paysage virtuel anamorphosé des péplums de la génération 2010 ou des séries alignées sur cette esthétique? Nous montrerons, dans un second temps, combien l'usage des ornements antiques est lié, dans la série Rome, à l'apparition des scènes paysagères de sorte que le paysage naturel, pour fonctionner comme marqueur antique, doit être mis sur le même plan esthétique que ces ornements antiques : c'est précisément la sérialité, intradiégétique (à l'intérieur d'un même épisode) et extradiégétique (entre les épisodes) qui permet cette mise en relation. 


\section{Nature de l'Antique, nature du paysage : le congé du panorama}

6 Dans les deux séries Atlantis et Odysseus, série à budget moindre que Rome $e^{15}$, la mise en scène du paysage antique passe par un métissage systématique entre un cadre naturel réel, celui du lieu de tournage - Maroc et Pays-de-Galles pour Atlantis et Portugal pour Odysseus - et reconstitution virtuelle. C'est au travers d'un jeu sur les proportions entre les deux composantes naturelle et virtuelle et surtout selon quel modus operandi la 3D est confrontée ou insérée dans le paysage naturel que se dessinent deux lignes de réception de la matière antique. Toutes deux opposées, elles ont cependant pour même conséquence de mettre à distance le schème dominant de représentation picturale du paysage : le panorama ${ }^{16}$.

7 La série proposerait-elle une approche paysagère de l'Antiquité par une esthétique singulière en « réaction » contre les modèles visuels dont elle s'inspire pourtant? Nous allons voir comment, le passage au petit écran de l'univers mythologique et épique de l'Antiquité, se traduit par la création d'un paysage plus modeste, plus intimiste, plus proche du spectateur contemporain ${ }^{17}$.

\section{Le paysage antique des séries ou l'essoufflement de la maximisation virtuelle}

8 Au premier abord, la série Atlantis semble s'inscrire dans l'air du temps : elle propose une mise en scène paysagère de l'Antiquité qui répond au processus esthétique de réception contemporaine de cette dernière, à savoir celui du schème de la «maximisation » du paysage urbain et naturel. Ce phénomène a été observé non seulement à propos de l'univers vidéoludique mais encore à propos des péplums hollywoodiens de la génération 2000-2010 et a été qualifié de «syndrome Acropole $\aleph^{18}$. Dans Atlantis, les paysages naturels comme urbains sont tous construits sur la même logique du grandiose, l'effet de maximisation passant par l'insertion d'éléments virtuels dans un paysage naturel déjà imposant: falaises abruptes (naturelles) qui soutiennent les imposantes murailles (virtuelles) de la cité d'Atlantis dès le tout premier épisode de la saison 1 [fig.1], montagnes élevées (naturelles) dont la hauteur est rehaussée par un effet de cadrage où le personnage - héros devant mener une quête dans cet espace sauvage et inquiétant - est filmé de dos (et inséré selon la technique de l'incrustation), avec un léger effet de contreplongée en train d'observer le paysage [fig.2] (procédé que l'on retrouve tout au long de la série), grottes profondes, virtuelles pour celles qui tiennent lieu de labyrinthe lors de l'affrontement du Minotaure dans l'épisode 1 de la première saison ou celles qui mènent au Tartare cachant la boîte de Pandore dans l'épisode 9 de la même saison [fig.3] et naturelles (filmées en extérieur et ensuite reconstruites en studio pour le jeu des acteurs) pour celles qui tiennent lieu de cachette aux brigands et aux Érinyes dans l'épisode 8 ou aux lépreux [fig.4]. Parmi ces paysages divers, peu sont gratuits : soit ils servent de plan de coupe soit ils sont le théâtre des aventures rocambolesques du trio de héros Jason, Hercule et Pythagore. 


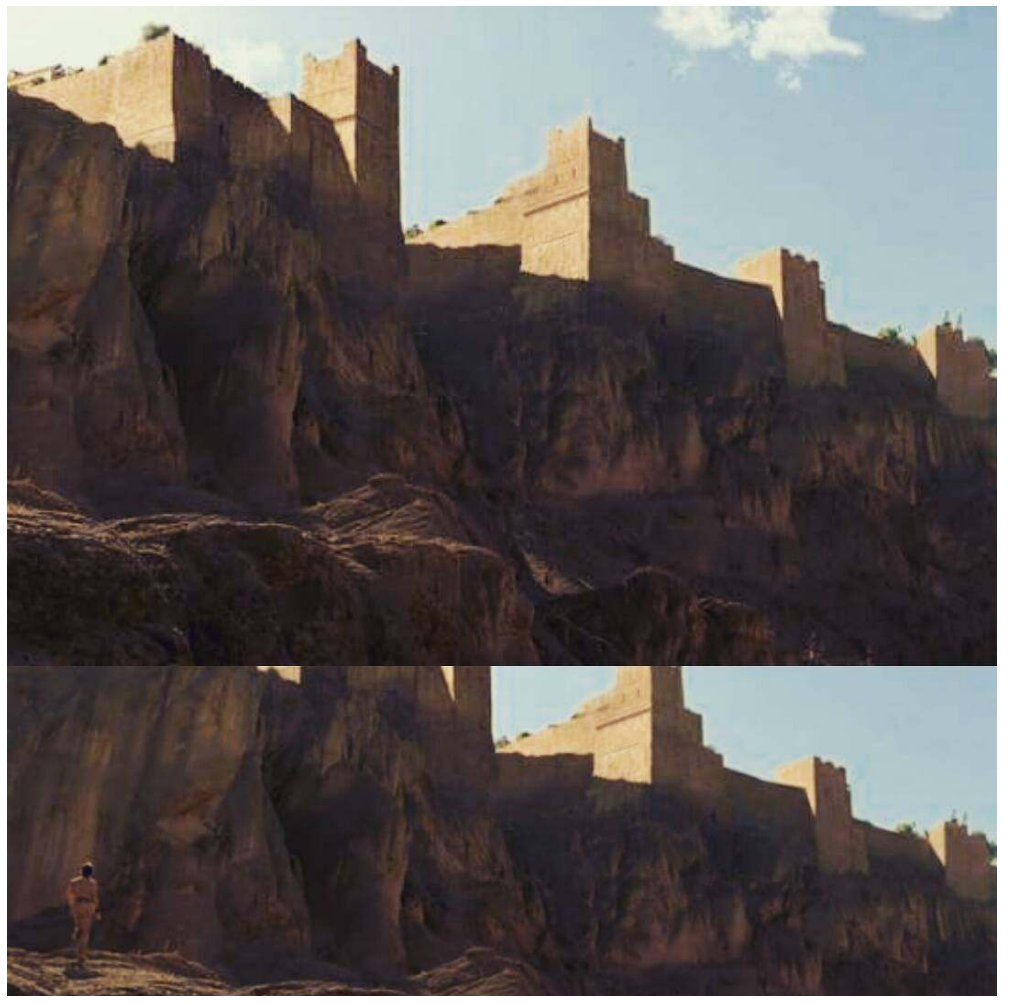

Fig. 1

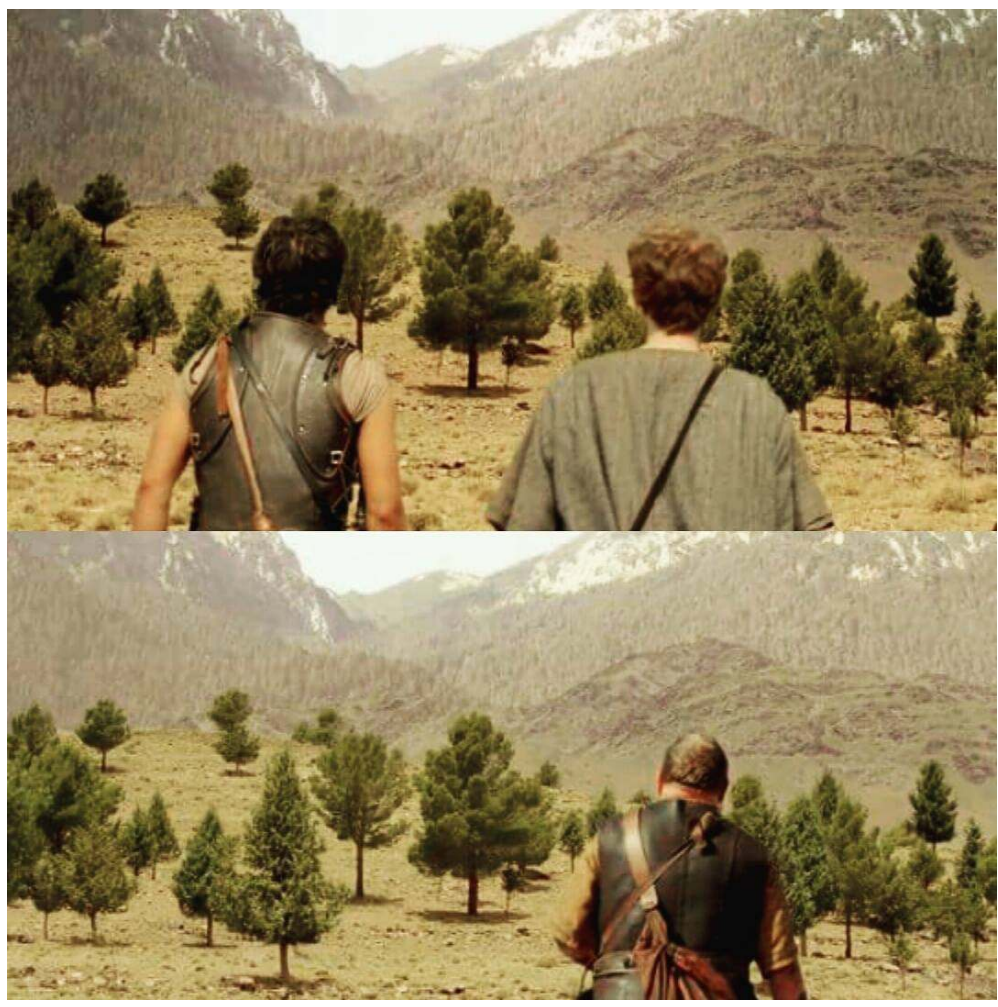

Fig. 2 


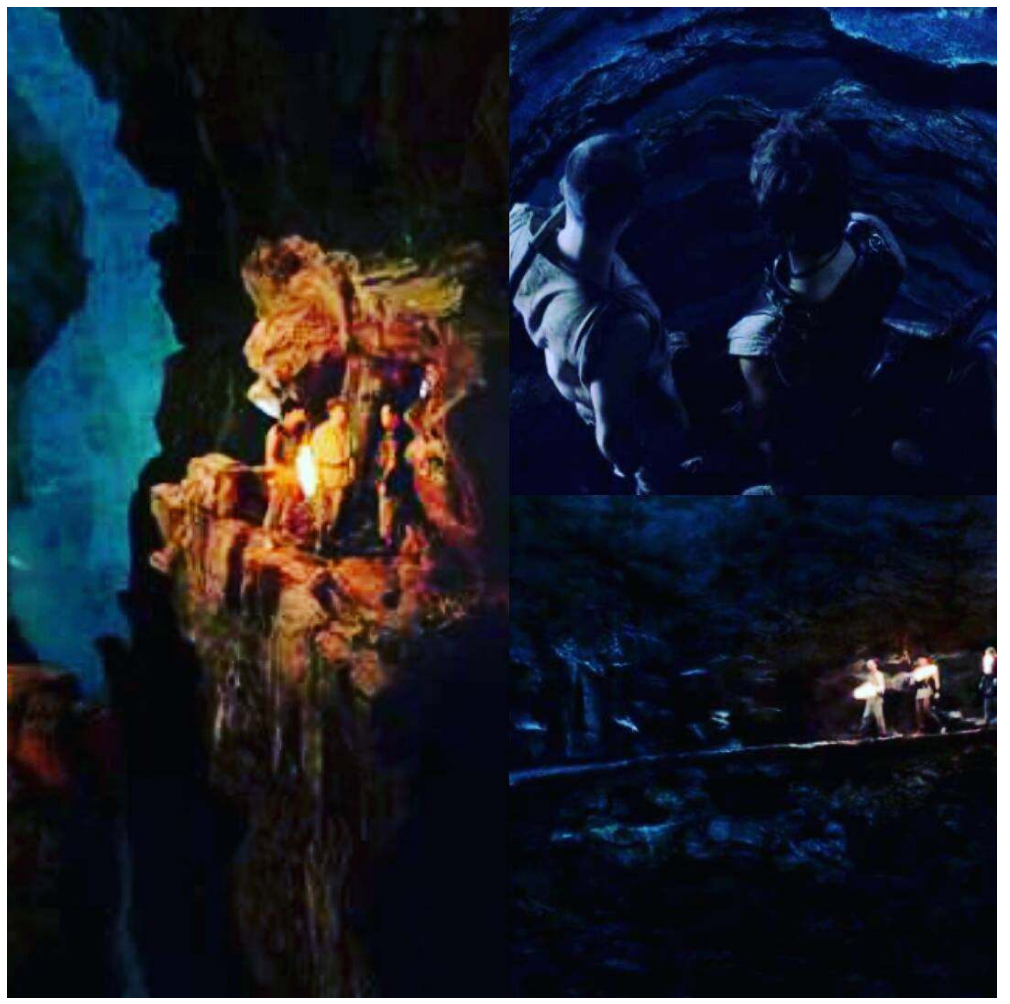

Fig. 3

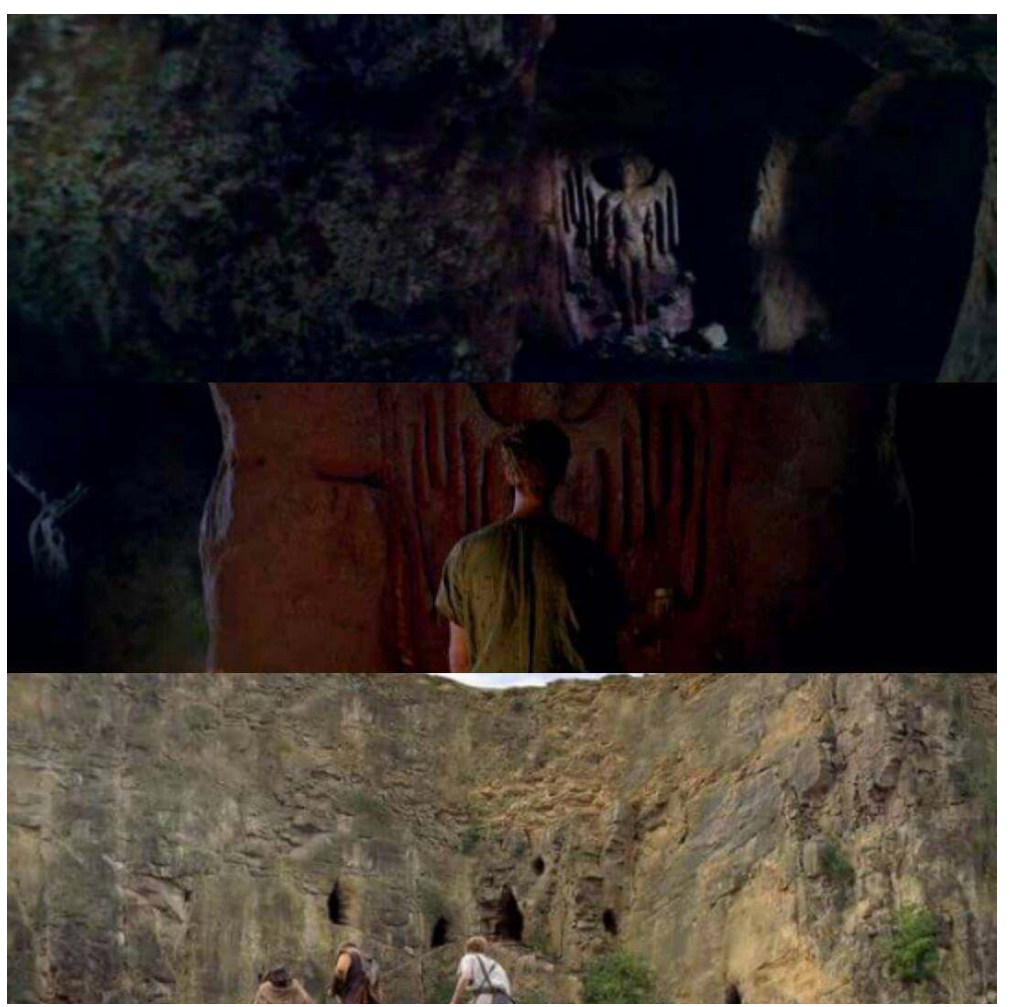

Fig. 4

9 L'épisode le plus emblématique étant sans doute celui consacré aux Érinyes (épisode 8, saison 1) et à la caravane dans le désert, dont les séquences ont été tournées au Maroc [fig.5]. 


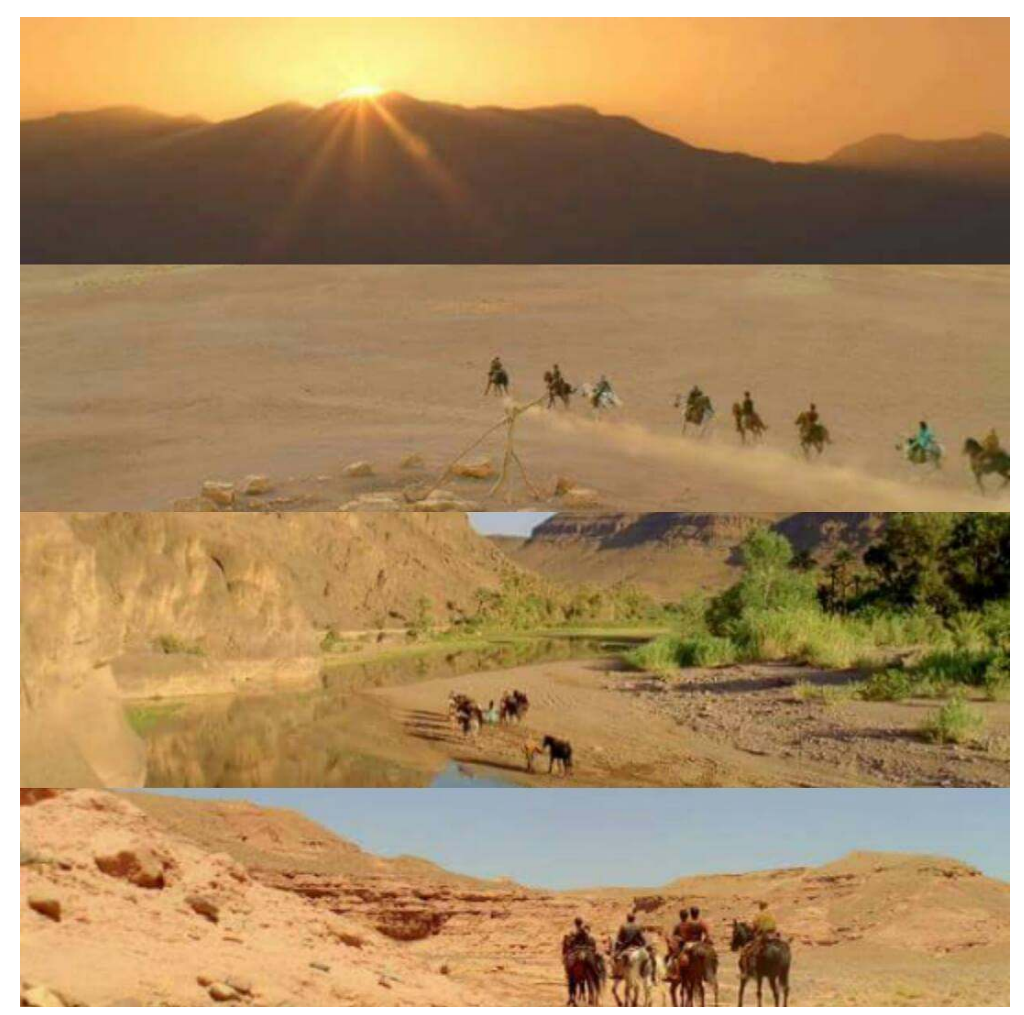

Fig. 5

10 Malgré ces beaux paysages naturels, malgré le souffle épique que la chevauchée des héros peut amener et malgré les effets de plans larges qui viennent appuyer le tout, on ne peut parler ici de panoramas grandioses: à chaque plan large qui montrerait le paysage désertique et la ligne d'horizon perdue, à chaque échappée qui construirait une dynamique spatiale horizontale à l'infini, correspond, dans la séquence, un plan qui dessine systématiquement une dynamique spatiale contraire, par la verticale. Les vents du désert, en spirale ascendante [fig.6] - véritable théophanie des Érinyes - créent non seulement une rupture à l'écran au sein de la logique spatiale et paysagère de l'épisode pensé sur le modèle horizontal de l'étendue désertique, à savoir au niveau intradiégétique, mais encore harmonisent, au niveau extradiégétique, la logique spatiale et paysagère de cet épisode avec l'ensemble de la série dominée par le paradigme de la maximisation verticale. 


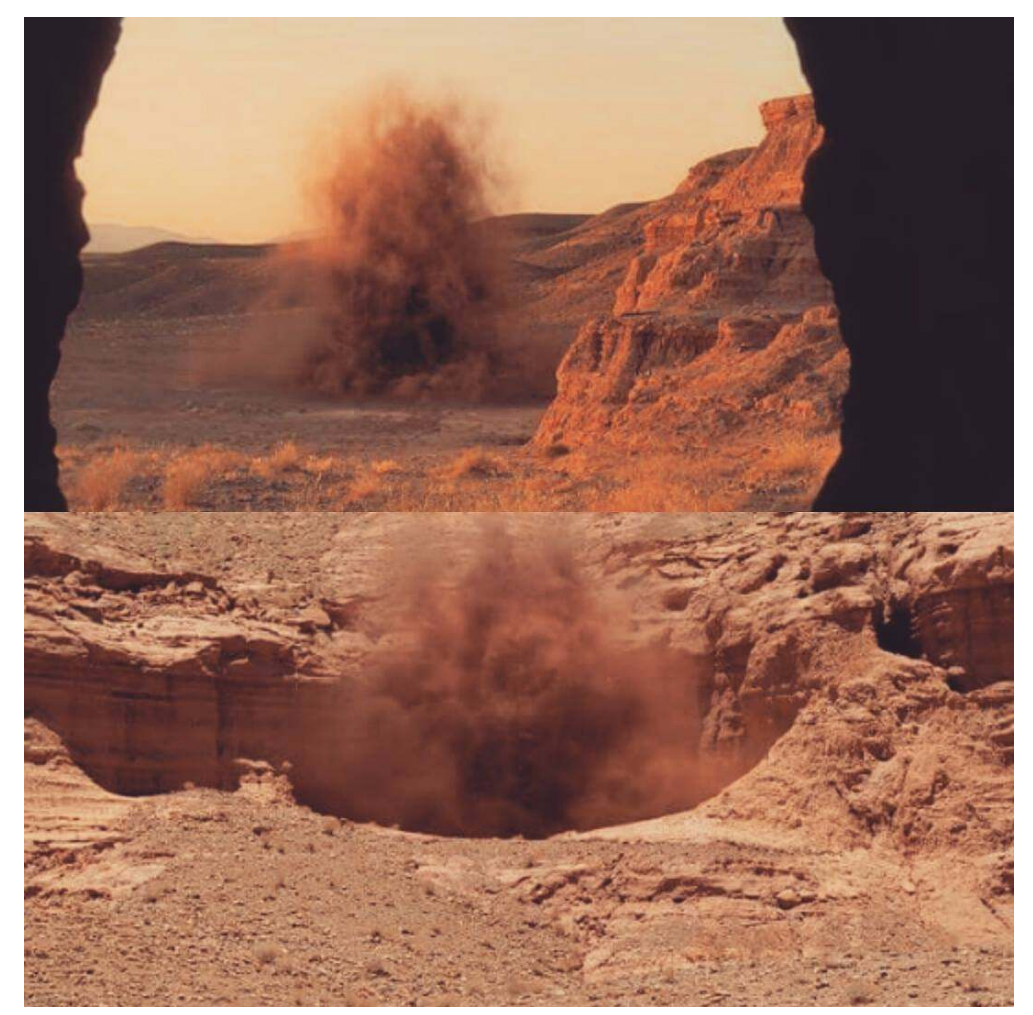

Fig. 6

11 Le plan qui clôt l'aventure de la caravane dans le désert nous le montre : l'incrustation virtuelle, en plein cadrage du paysage naturel, d'une ville du désert dont la grandeur et la hauteur est telle qu'elle déborde le cadre, répète encore le paradigme de l'hypertrophie paysagère à la verticalité dominante [fig.7].

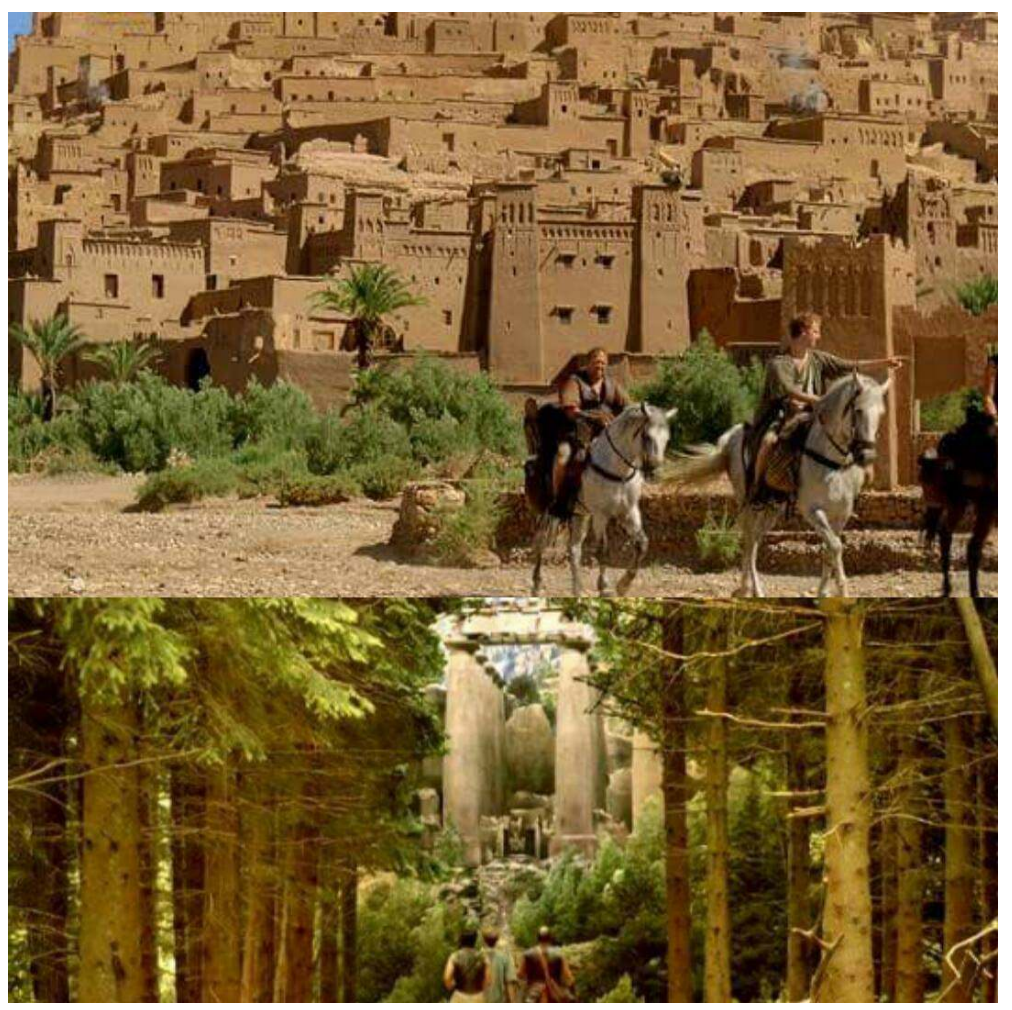

Fig. 7 
12 des jeux vidéo ou des péplums hollywoodiens, il faut tout de même noter que le spectateur est confronté à un paysage à dominante naturelle dans lequel vient, de temps à autre, se greffer l'hybridité d'une 3D maximisante mais dont la présence est modeste, presque « raisonnée ». lui-même imposant, comme le temple où se cache le frère de la princesse Ariane (épisode 5 de la saison 1 [fig.7]), c'est l'espace urbain qui emblématise le mieux cette technique de la maximisation paysagère par la verticale. À de nombreuses reprises dans la série, au rythme d'un plan par épisode quasiment, apparaît au spectateur un bel exemple du «syndrome Acropole $~^{19}$. Un montage présente à l'écran les véritables soubassements de l'Acropole d'Athènes et sa structure extérieure, incrustés dans le décor de studio. La composition de l'image dont la vue reprend un vue célèbre de l'Acropole depuis l'une des rues du quartier Plaka, fort fréquenté et fort touristique à l'heure actuelle [fig.8], nous montre de nouveau que, bien qu'elle soit construite en hauteur (bien plus haute que l'Acropole athénienne dans la réalité), c'est toujours le paradigme du «naturel » et du vraisemblable qui domine, c'est celui du quotidien du spectateur: le «cadrage touristique " de cette Acropole d'Atlantis, reprenant celui de l'Acropole d'Athènes actuellement en est sans doute l'exemple le plus convaincant.

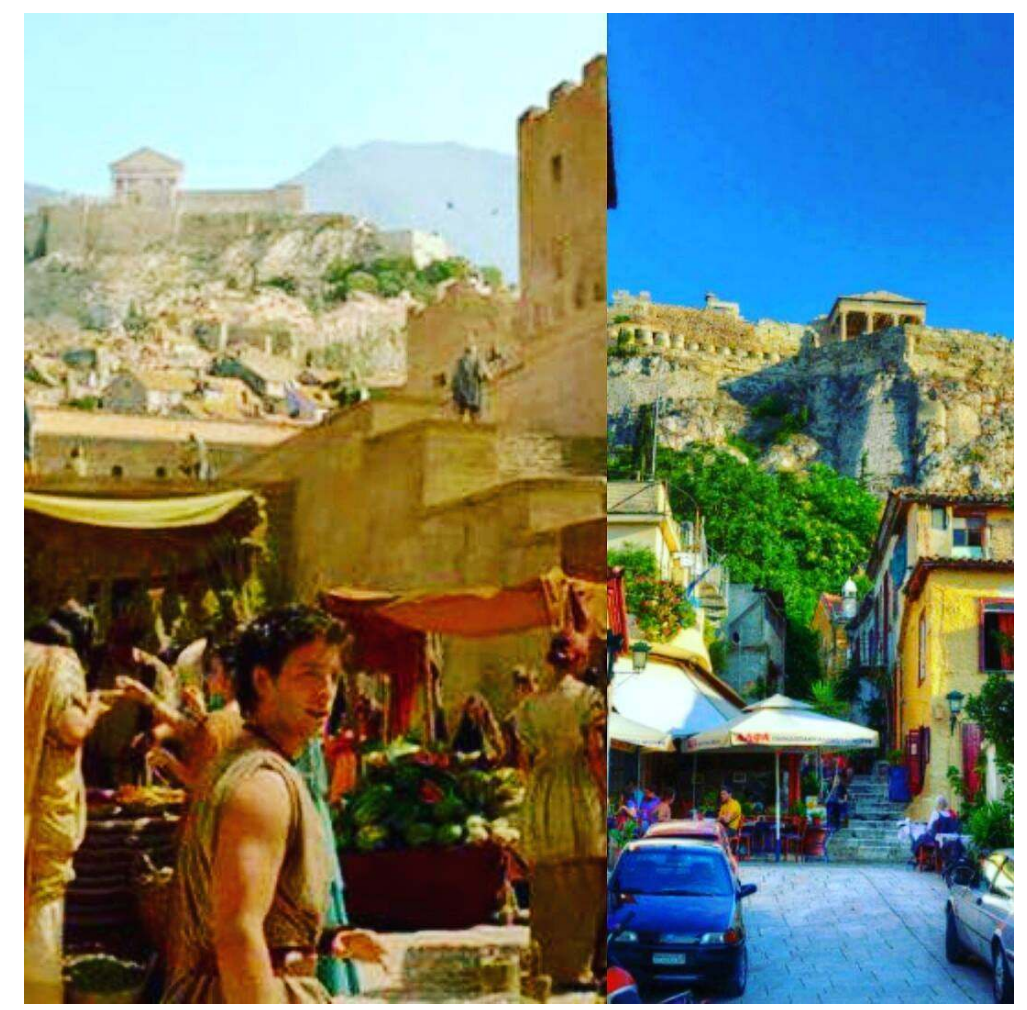

Fig. 8

14 Cette prédominance de l'Acropole dans la série n'est pas fortuite: elle organise les déplacements des héros et joue un rôle fondamental dans la diégèse au point qu'elle constitue non seulement le point centrifuge et centripète de l'ensemble du paysage de la série et qu'elle structure les épisodes entre eux et en eux-mêmes ${ }^{20}$. 


\section{Insertion virtuelle, retour à l'archaïque et intimité}

15 Ce resserrement et cette dilatation autour de l'Acropole d'Atlantis - construite sur le modèle athénien - ne va pas sans créer un effet de huis-clos de plus en plus exploité dans la série : les intrigues de palais se multiplient au fil du déroulement des épisodes, les plans en intérieur et en extérieur sont resserrés sur les héros, la dimension psychologique et morale intervient de plus en plus lorsque les personnages sont cadrés seuls ou à deux, sur fond de nature sauvage ou sur fond de décor antique : la construction du paysage antique, naturel ou urbain, passe désormais par le relais des personnages à l'écran, par leurs états d'âme ou par la symbolique des décors antiques qui occupent une place prépondérante dans la saison $2^{21}$. Ce resserrement sur le point nodal de l'île, fait adhérer la logique paysagère de la série à la représentation archaïque du monde selon les anciens Grecs et dessine ainsi une poétique de l'humilité des âges archaïques et de l'intimitéé ${ }^{22}$. De nombreux épisodes présentent des espaces forclos comme les grottes, le labyrinthe, déjà évoqués précédemment, les souterrains du palais où est caché le Palladion (épisode 1 et 2 de la saison 2) ou encore les couloirs dérobés conduisant de la chambre d'Ariane au temple de Poséidon, le naos du même temple où l'oracle officie... tous ces espaces renvoient à la logique de la forclusion, du repli et de l'intimité, en harmonie avec la représentation archaïque du paysage insulaire et primordial. Tous ces espaces du repli et de la forclusion confrontent les héros à leur destin et les renvoient systématiquement à leur intériorité: c'est l'épreuve de soi-même, de son identité, de ses responsabilités comme en témoigne la difficile épreuve du pardon dans l'épisode des Érinyes (8, saison 1).

16 Ainsi, le schème paysager du panoramique se voit congédié au profit de celui de l'insularité, de la forclusion et de l'intimité. La série Atlantis, bien que s'inscrivant apparemment dans la ligne dominante de l'esthétique grandiose du paysage antique, contribue à l'émergence d'une autre ligne de réception du paysage antique dans les séries des années 2000-2010, qu'Odysseus vient parachever en proposant un nouveau paradigme paysager, celui du sentiment nostalgique.

\section{Nostos et saudade dans Odysseus, un nouveau paradigme paysager.}

17 Cette série ${ }^{23}$ offre, bien plus qu'Atlantis, une utilisation de la 3D restreinte au profit d'une mise en scène du paysage réel ${ }^{24}$. Une ligne de réception épique différenciée se fait jour ici : elle détache Odysseus de l'épique "grandiloquent » du cinéma et des jeux vidéo qui lui sont contemporains. De manière systématique, cette série propose un resserrement de l'épopée autour du quotidien des héros, montrés dans leur solitude psychologique, c'est un "épique du quotidien ${ }^{25}$, une expérience de l'humilitée ${ }^{26}$. Un des paysages les plus propices à ce resserrement sur l'intimité des personnages est le rivage d'Ithaque [fig.9]. 


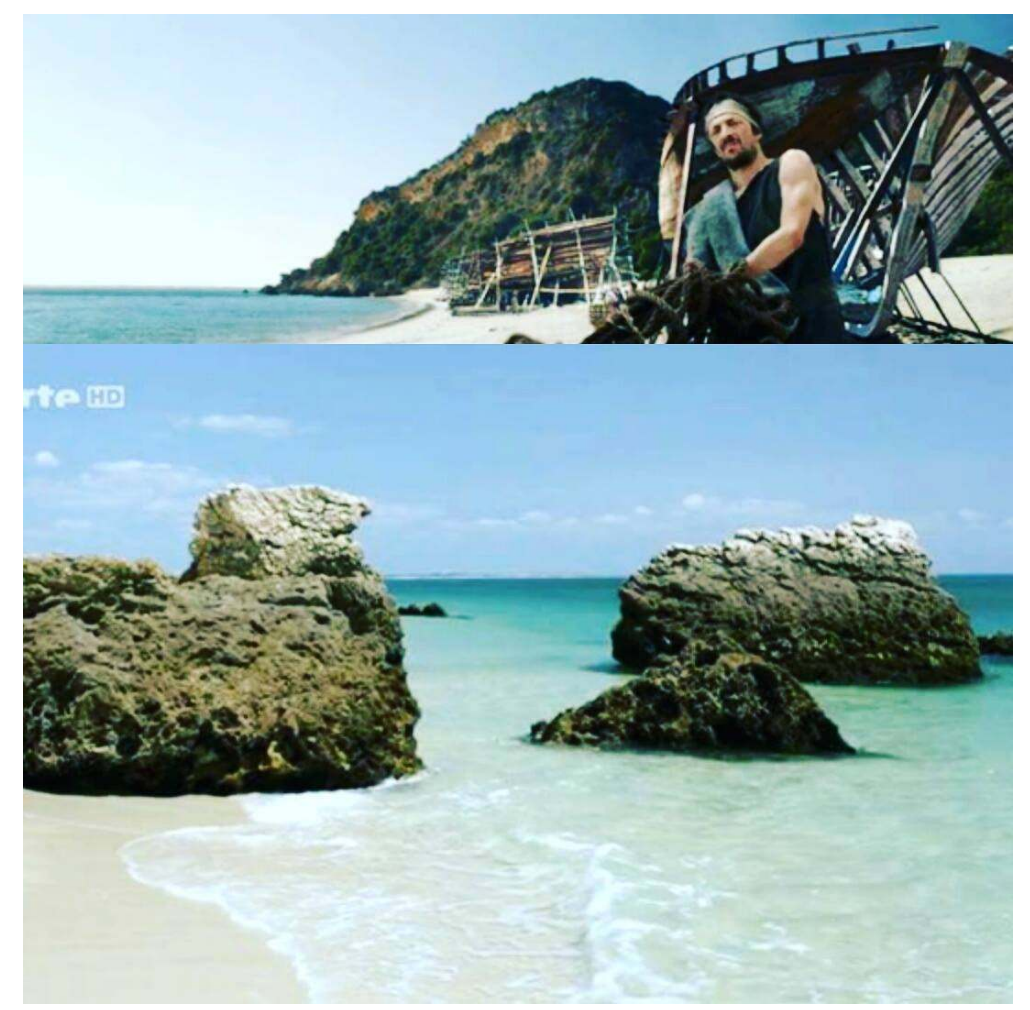

Fig. 9

L'épisode 7 propose un plan d'Ulysse, de dos, en plein récit de son périple à Orion [fig. 10].

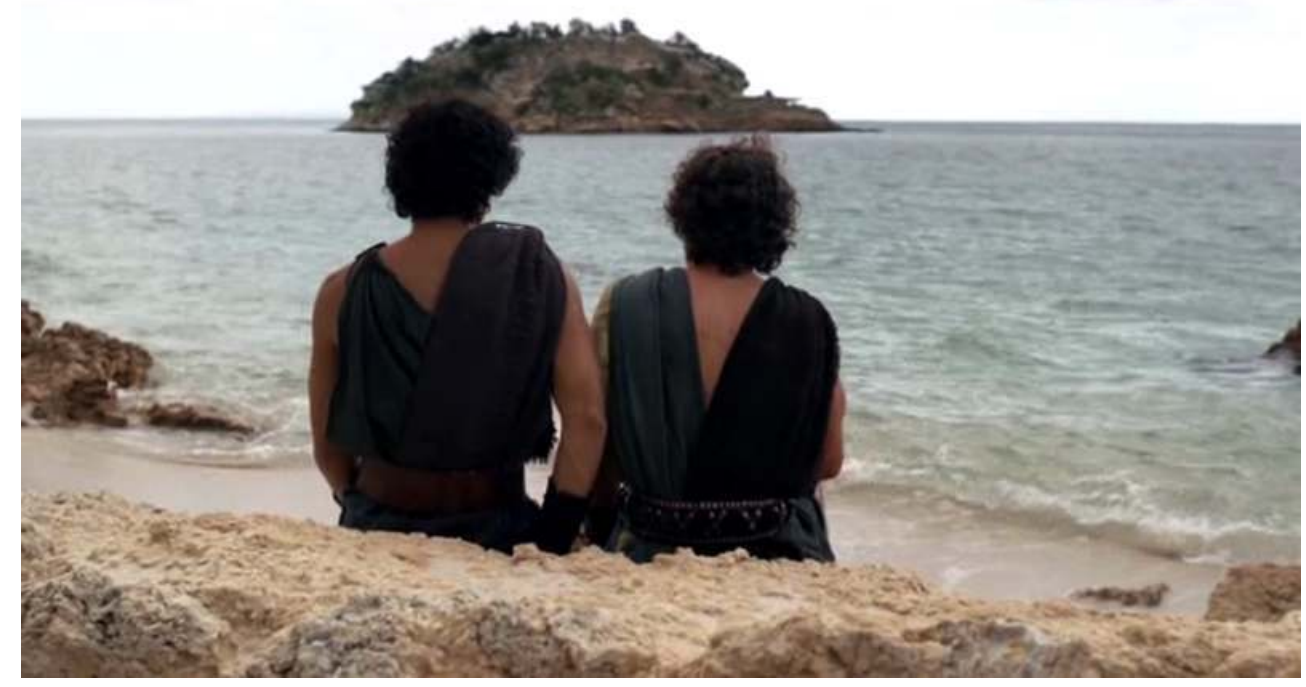

Fig. 10

Ce plan montre que le voyage d'Ulysse, qui constitue la part la plus importante du récit homérique ainsi que l'horizon d'attente « canonique » n'est pas totalement absent de la série. Il y est, de manière très pertinente, sans cesse renvoyé à distance, repoussé dans un passé diégétique qui le résorbe et le contient ${ }^{27}$. Cette mise à distance peut aussi se lire sur un plan méta-poétique, ou méta-filmique, comme ce qui repousse l'épique « classique »dont le schème du panoramique souligne la grandeur - en permettant l'émergence d'une autre modalité de l'épos à l'écran, celle du nostos et de la saudade. 
20 Nombreux sont les plans de la série qui prennent place sur la grève d'Ithaque, ou bien montrent, de loin ou à travers des dispositifs spécifiques, des vues du rivage. C'est le cas des plans filmés à travers la fenêtre à claustra de Pénélope [fig.11], qui scrute chaque jour le rivage, espérant le retour d'Ulysse.

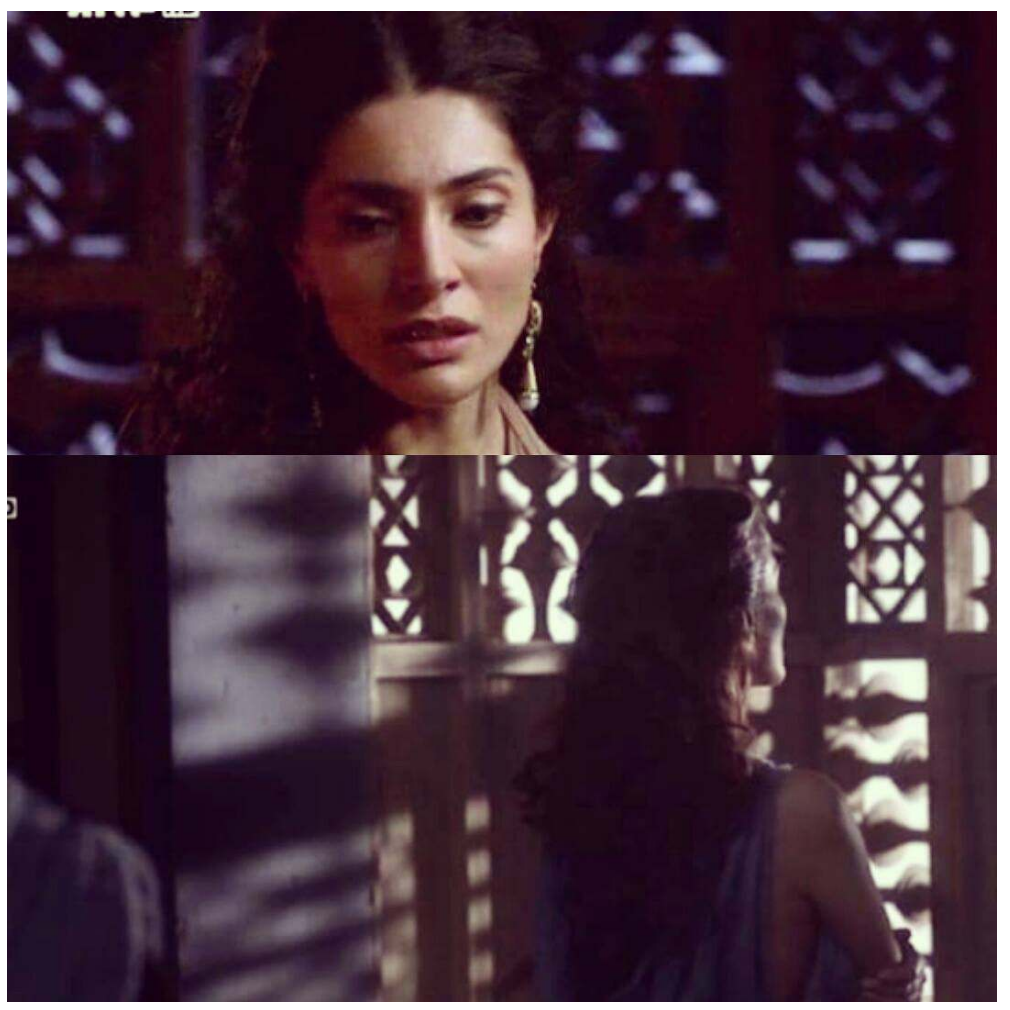

Fig. 11

21 Le nostos ${ }^{28}$ est bien présent dans Odysseus, son actualisation singulière passe par une mise en scène singulière du paysage du rivage. Il est le cadre du retour d'Ulysse : à la fin de l'épisode 4, Ulysse est découvert par Thyoscos le devin, inconscient, échoué sur la grève, encore attaché au mât brisé de son navire [fig.12], après - c'est du moins ce que nous supposons par inférence - l'épisode des Sirènes qui a vu le vaisseau se briser entre les roches Planktes. 


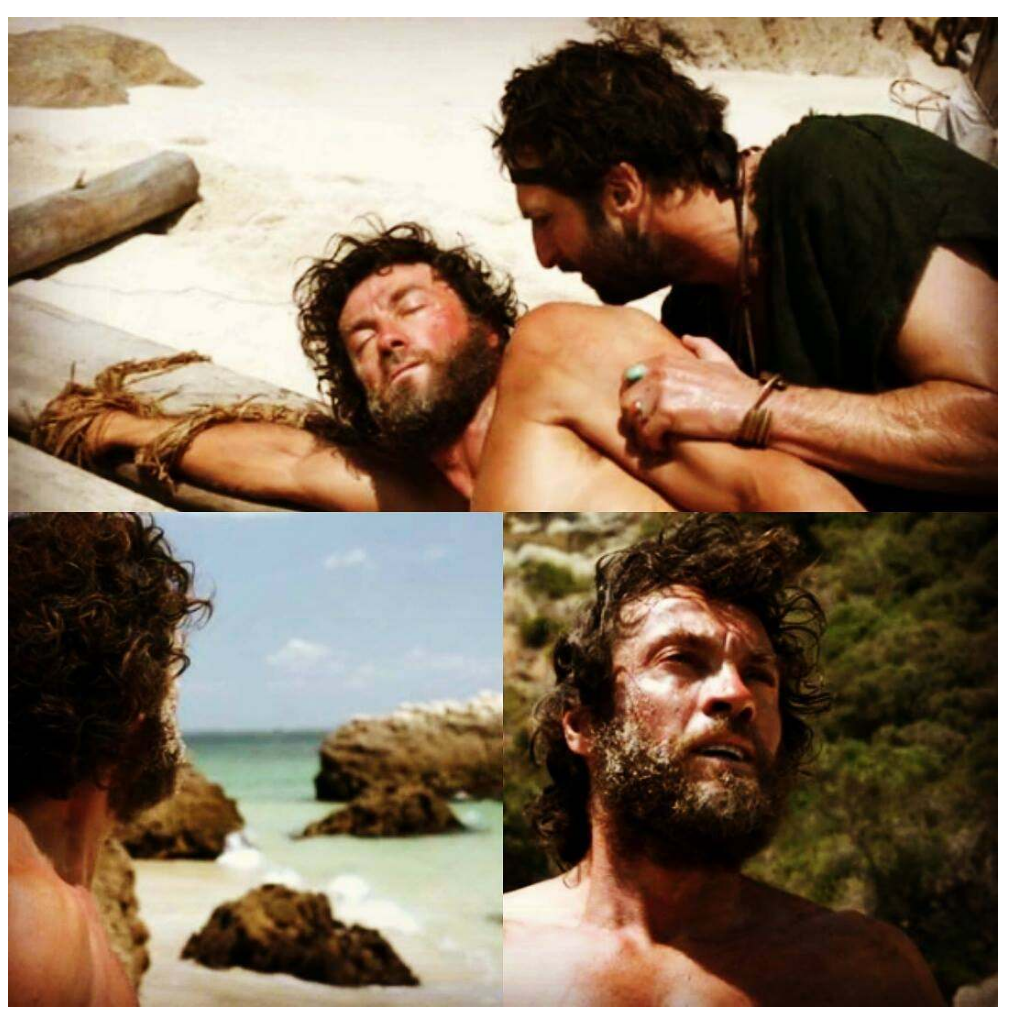

Fig. 12

22 Toutefois, lorsque Ulysse, aidé par Thyoscos, se relève, et qu'il est reconnu par ce dernier, ce n'est pas sur Ithaque que se porte son regard, mais sur l'horizon méditerranéen. Ce regard participe dans la série d'un mouvement plus large : il semble qu'Odysseus propose une inversion de ce que l'on entend habituellement comme la poétique homérique du nostos. Ici, ce n'est pas tant la nostalgie du retour vers le foyer, que celle du voyage qui est mise en scène par le paysage littoral. Ce retournement du nostos du doux foyer originel en nostos du voyage est, selon Barbara Cassin ${ }^{29}$, déjà inscrit dans les textes possibles ouverts par la narration homérique. Comme la philosophe le fait subtilement remarquer : à peine rentré, Ulysse doit à nouveau quitter son épouse Pénélope, au lendemain même de la nuit de leurs retrouvailles. Il doit repartir pour accomplir, afin d'apaiser la colère de Poséidon, un dernier exploit ${ }^{30}$. Le rivage - dans une remotivation de sa fonction ${ }^{31}$ - incite Ulysse à la remémoration des épisodes de son périple et à se laisser aller à la nostalgie. Une nostalgie qui, curieusement, prendrait des résonances portugaises: les scènes de la série étant tournées sur le rivage atlantique à $30 \mathrm{~km}$ de Lisbonne, évoqueraient alors la saudade, en lieu et place du nostos épique grec. Le désir du " plus lointain du lointain », formulé ici en termes de douceur, se superpose à ce subtil mélange de nuances émotionnelles, propre à la culture portugaise, défini comme "bonheur hors du monde » par Luís de Camões. Il rencontre alors, à la manière d'un heureux kairos, nos enjeux d'un épos de l'humilité, dans la mise en scène paysagère qu'en propose Odysseus, en montrant à l'écran comme du paysage méditerranéen odysséen, ce qui est dans la réalité physique un rivage atlantique portugais, tourné vers le Nouveau Monde et non vers le mare nostrum ${ }^{32}$. Ainsi, c'est la création d'un espace tiers, d'un nouveau paysage épique, fait d'une remotivation mutuelle des espaces de ces deux prismes culturels à laquelle assiste le spectateur. L'épos à l'écran dans la série Odysseus peut alors se comprendre comme un congé du panoramique permettant l'émergence du schème de l'intime, autre possible paysager dont la sérialité répète la douceur et l'humilité, comme le nostos ne cesse de s'emparer du cœur d'Ulysse, 
comme la saudade ne cesse de résonner aux oreilles des lusitanophones. Retour incessant, figure de l'éternité : la série Rome célèbre l'éternelle ville-monde de l'Antiquité au travers d'une dernière modalité esthétique qui oppose ou complète le schème paysager du panoramique en lui confrontant celui de l'ornement.

\section{Rome, la ville-monde, l'ornement-paysage}

Avons-nous encore besoin de présenter la série d'HBO consacrée à un moment-clef de l'histoire romaine ${ }^{33}$ ? Parue dès 2005 , elle a su tenir le difficile pari d'intéresser et de séduire un large public - pour la plupart néophyte - avec un sujet qui pourrait être sinon rébarbatif, du moins réservé à quelques passionnés ou spécialistes d'histoire antique. Bien que qualifiée de péplum par l'ensemble de la critique, cette série présente un scénario et un traitement du paysage et des décors qui nous font hésiter à plus d'un titre sur le genre auquel elle appartient ${ }^{34}$. Répondant au regain d'intérêt pour les mises à l'écran de l'Antiquité dans la décennie 2010, cette série se démarque tout à la fois de la ligne de réception de la matière antique dessinée par les péplums de la génération 2000 et de celle des péplums et des jeux vidéo des années 2010. Non seulement Rome ne présente aucune ruine anachronique destinée à servir de chronotope antique comme on en trouve dans Troy de W. Petersen ou Alexandre d'O. Stone ${ }^{35}$, mais encore elle ne présente aucune utilisation de la 3D maximisante ${ }^{36}$, ni en milieu naturel ni en milieu urbain. Toutes les scènes en extérieur ont été tournées dans les paysages de l'Italie et dans les somptueux décors des studios romains de Cinecittà reproduisant le forum, les villas patriciennes et les bas-quartiers de la Rome tardo-républicaine et impériale ${ }^{37}$. Le paysage naturel actuel de l'Italie sert donc de paradigme à la mise en scène du paysage romain antique et ce, à côté de décors particulièrement soignés qui reproduisent avec minutie les villas patriciennes où l'on peut reconnaitre les grandes œuvres du répertoire pictural campanien ${ }^{38}$ comme la magnifique reproduction ornant le triclinium de la maison d'Atia qui reprend les fresques monochromes noires du triclinium de la Farnésine (Musée National Romain), ornées de paysages sacro-idylliques [fig. 13 et 14] comme les reprises, dans l'atrium de la maison d'Atia, des mégalographies de la célèbre fresque de la villa des Mystères de Pompéi [fig.15]. 


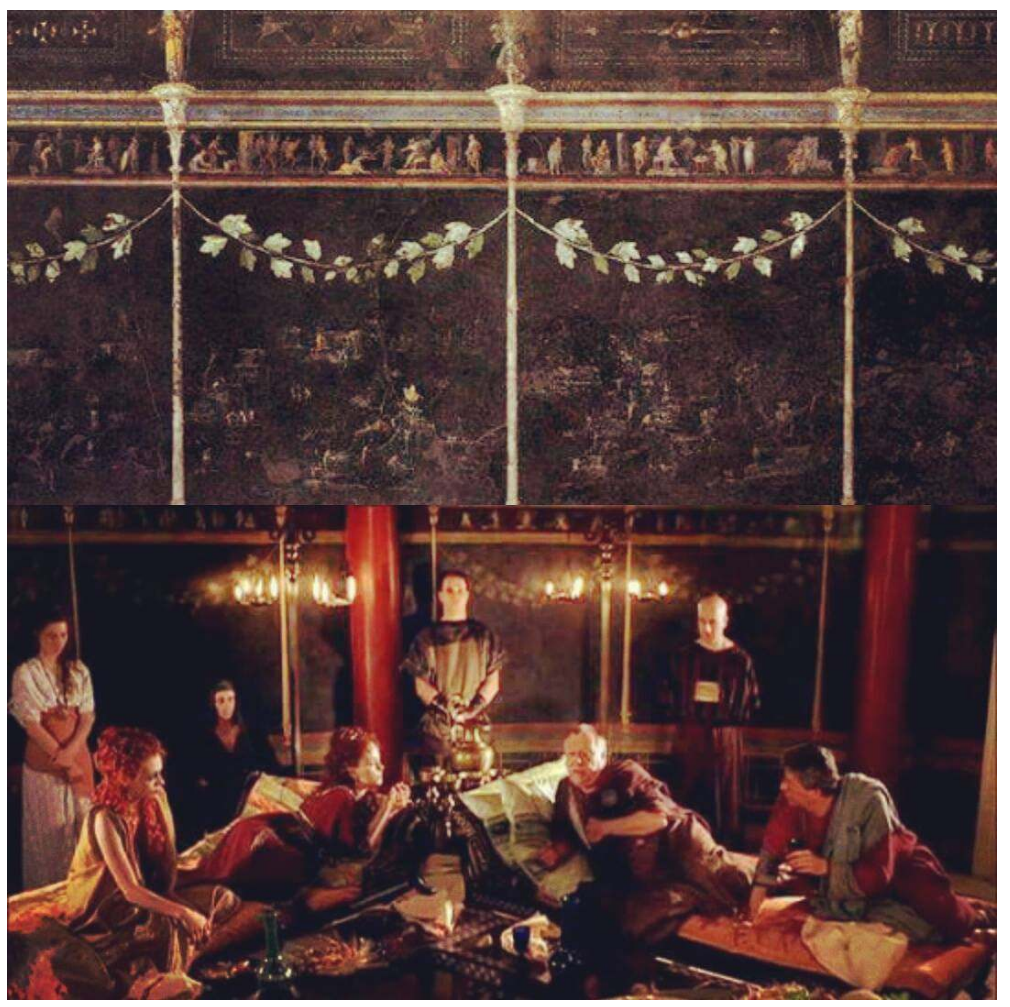

Fig. 13

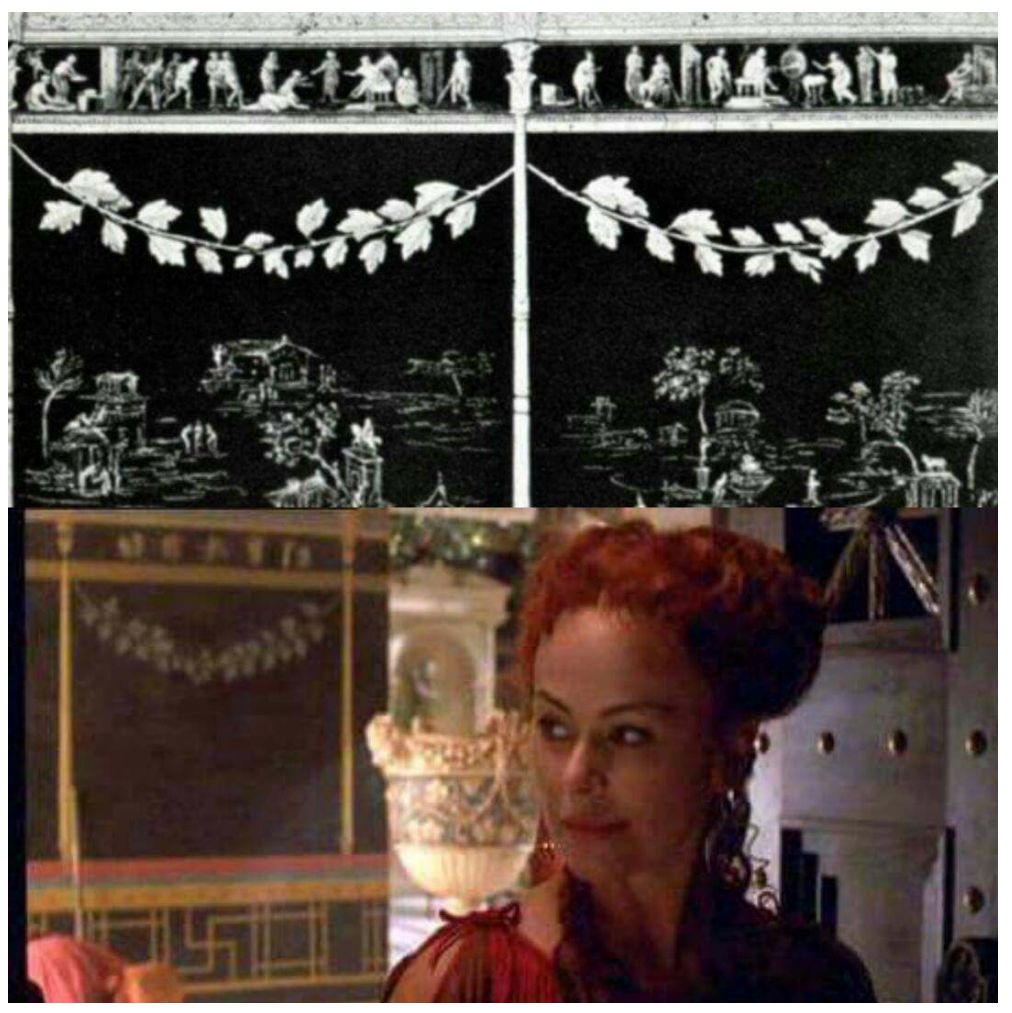

Fig. 14 


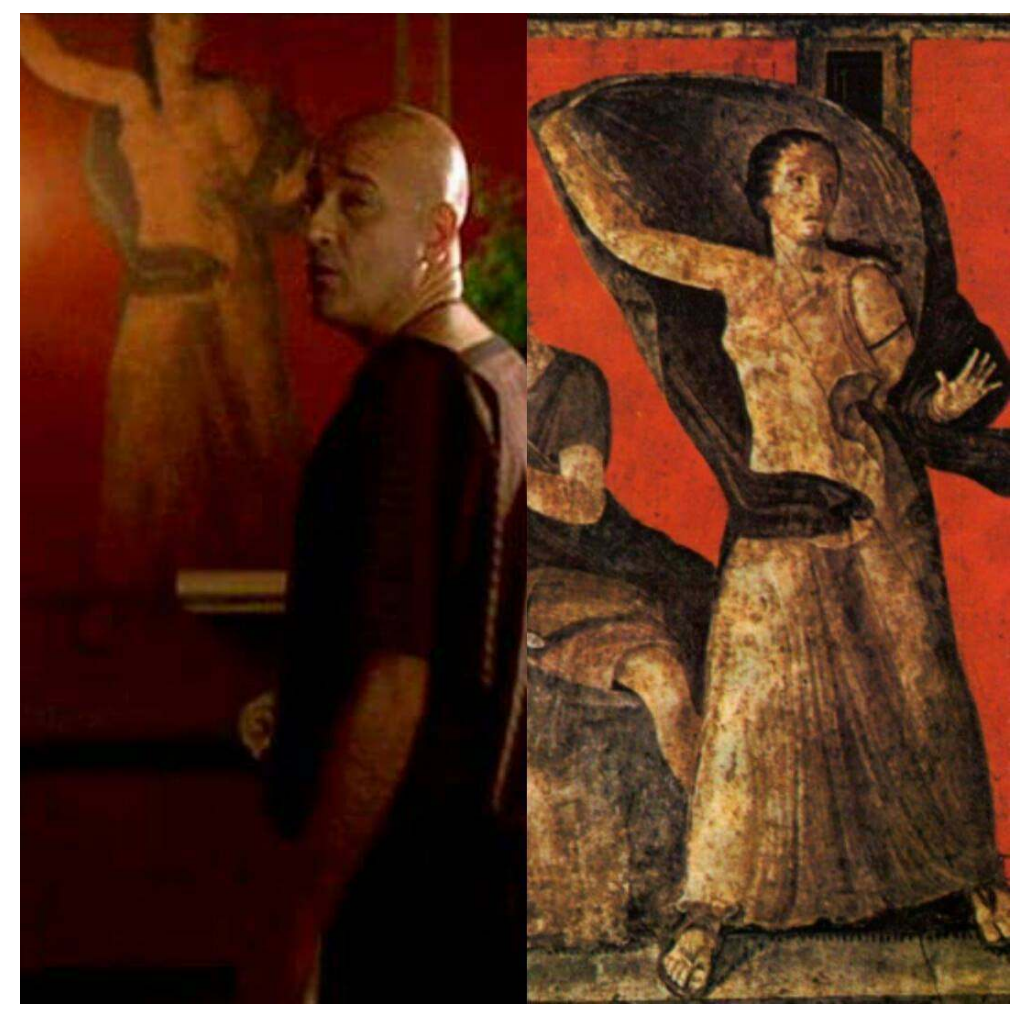

Fig. 15

24 Ainsi, aux côtés de ces décors vraisemblables dont la facture artistique reproduit la richesse des ornements du grand art pictural antique et de celle de la décoration intérieure, le paysage naturel réel et actuel semble courir le risque d'une certaine incongruité visuelle [fig.16 et 17].

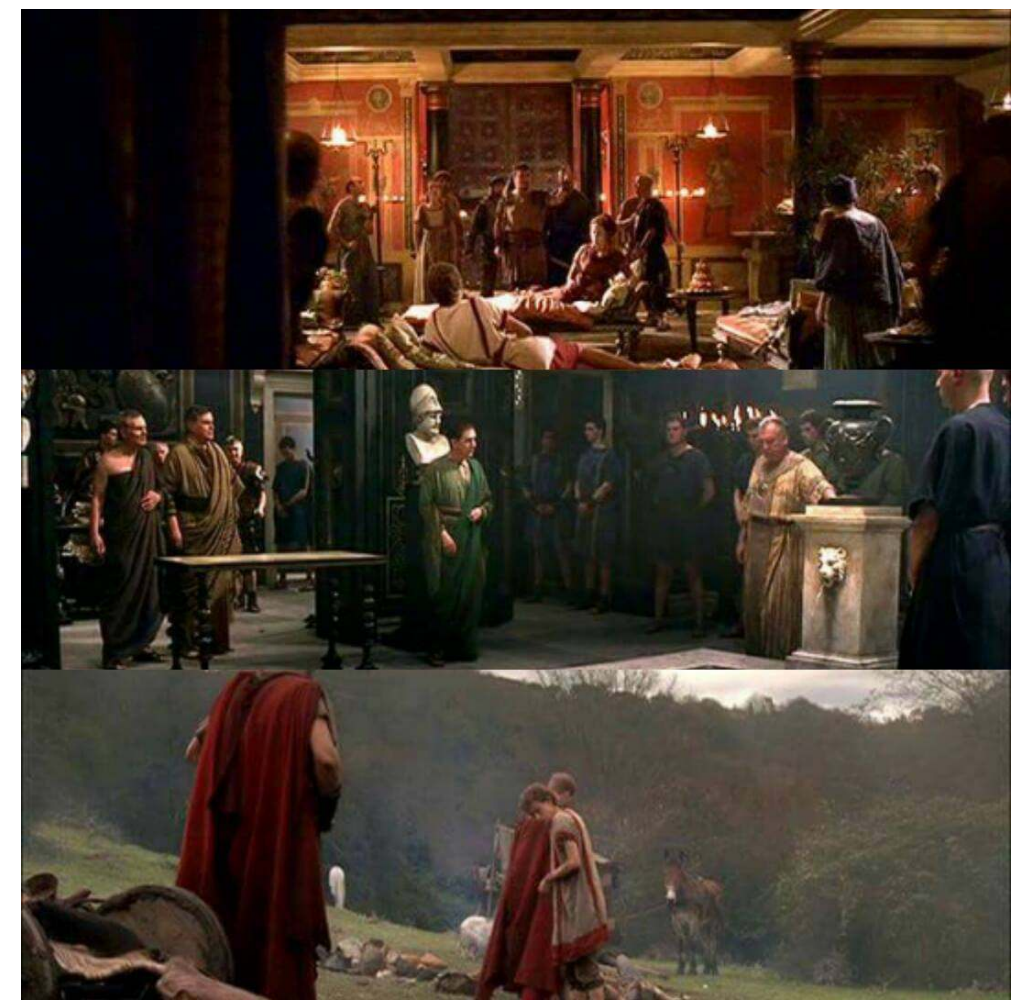

Fig. 16 


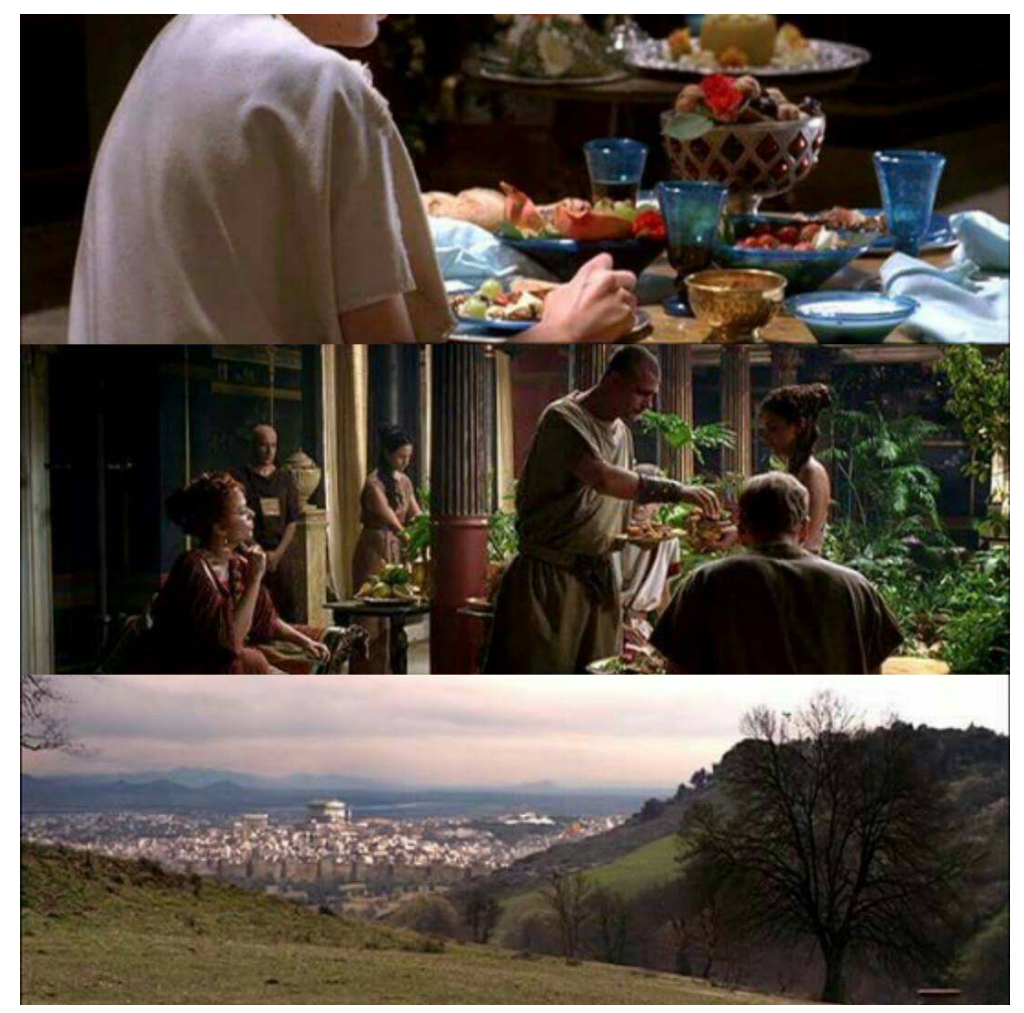

Fig. 17

Pourtant le principe de sérialité, propre à la logique générique de la série télévisuelle relie étroitement l'usage des ornements antiques ${ }^{39}$ à l'apparition des scènes paysagères de sorte que le paysage naturel, pour fonctionner comme marqueur antique, doit être mis sur le même plan esthétique que ces ornements antiques. Nous avons retenu deux cas particuliers de cette sérialité qui fonctionne tant sur le plan intradiégétique (à l'intérieur d'un même épisode) qu'extradiégétique (entre les épisodes).

\section{La ville-monde}

L'ensemble de la série Rome présente une harmonie de paysages et de décors. Si, dans la saison 1 l'essentiel des péripéties se déroule à Rome et dans la campagne du Latium ${ }^{40}$ - en dehors des épisodes qui traitent ponctuellement des campagnes militaires ${ }^{41}$ - et la saison 2 alterne les paysages urbains de Rome et ceux d'Alexandrie, on note cependant une organisation spatiale et narrative selon le même schéma: Rome reste le point centrifuge et centripète de l'ensemble de la série. Elle est le centre d'où partent toutes les aventures et vers lequel toutes convergent. Cette dynamique spatiale organise la diégèse à l'écran : le montage propre à chaque épisode, que ce soit la saison 1 ou la saison 2, fait régulièrement alterner séquences en paysage naturel plus ou moins éloigné de l'Urbs et séquences en paysage urbain, à Rome [fig. 18]. 


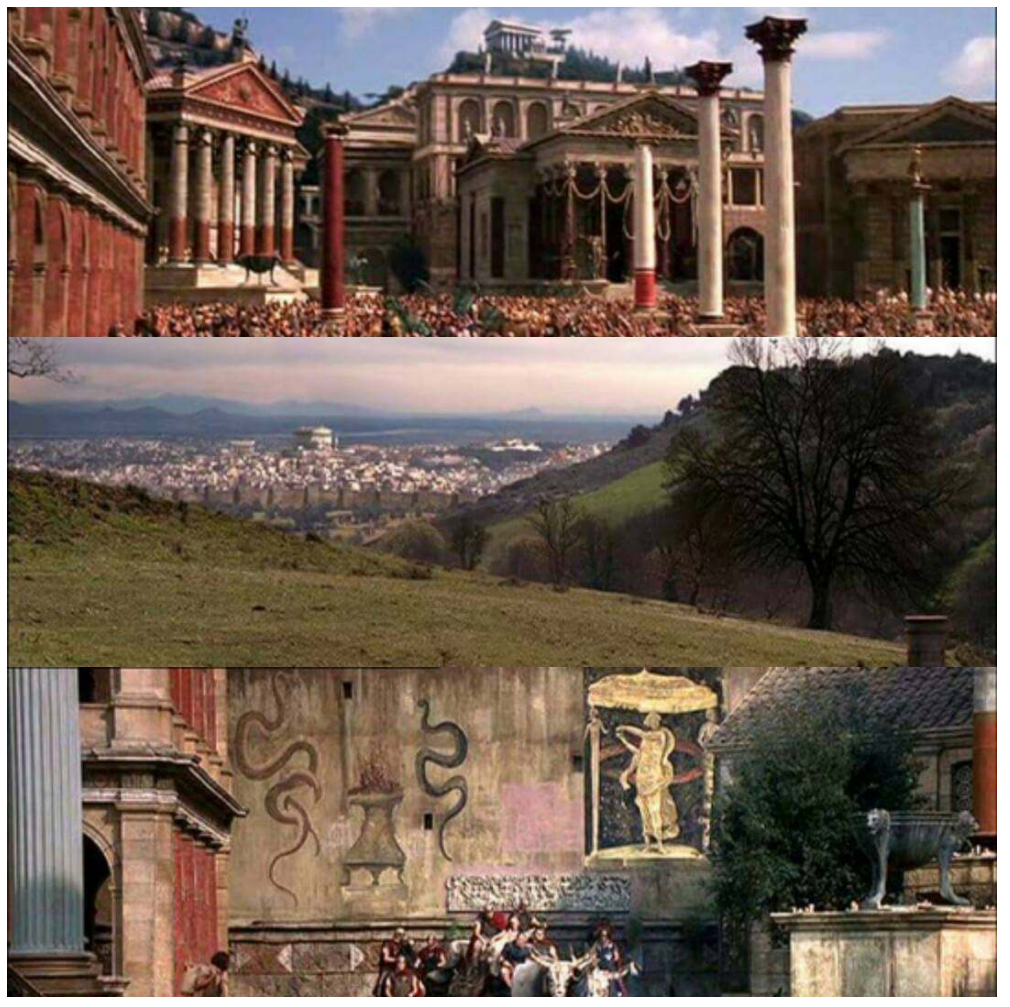

Fig. 18

Ce principe de resserrement et d'accélération de l'alternance des scènes paysage/ ornement organise également l'ensemble du déroulement de la série: le huis-clos se resserre sur l'espace intime des villas patriciennes des personnages principaux, Aitia, Pompée, Servilia (saison 1) puis Aitia, Marc-Antoine (et Cléopâtre), Octave pour la saison 2, donnant l'impression, avec le principe de sérialité que plus on avance dans la série, plus le spectateur devient l'intime, presque l'invité des grands personnages historiques portés à l'écran : cette impression est renforcée par la multiplication des scènes de banquet et de grandes soirées [fig. 19], notamment chez Aitia ainsi que par les scènes sexuelles où le spectateur assiste, en voyeur, aux intrigues d'alcôve qui sous-tendent le jeu politique, toile de fond sur laquelle se joue le destin de Rome, capitale et berceau de "notre » culture européenne ${ }^{42}$. 


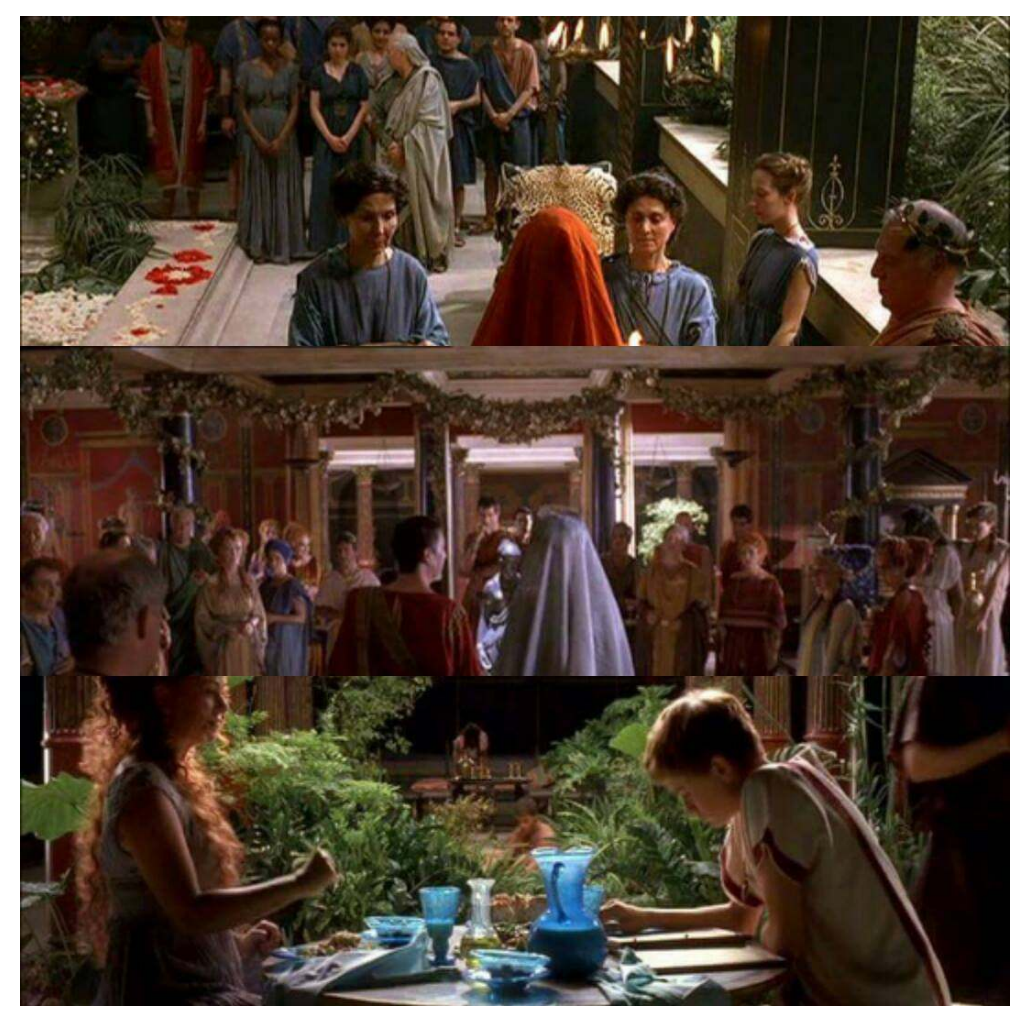

Fig. 19

Enfin, Rome dans cette série, bien que représentée à partir d'une excellente documentation sur le plan historique et archéologique, n'échappe pas à une certaine mise en scène stéréotypée: le Forum est surmonté du Palatin couvert d'un temple (anachronique) [fig. 18] - possible réminiscence du « syndrome Acropole » - il donne lieu à une vue sur l'arc de triomphe de Titus (anachronique [fig. 20 et 21]) lors de la scène de triomphe de Jules César, l'ensemble du corpus pictural sollicité pour orner les villas patriciennes est largement postérieur à la période tardo-républicaine, le palais alexandrin de Cléopâtre n'échappe pas au clinquant ${ }^{43}$ du faste hollywoodien remontant aux productions de Cecil B. DeMille [fig. 22 et 23], les ornements intérieurs - souvent inspirés des realia antiques analysés plus haut, témoignent tous de l'idée de faste et de richesse qui singularise l'esprit de la culture romaine dans notre mentalité actuelle et assure, à l'écran, une continuité aux épisodes entre eux et en eux-mêmes ${ }^{44}$. 


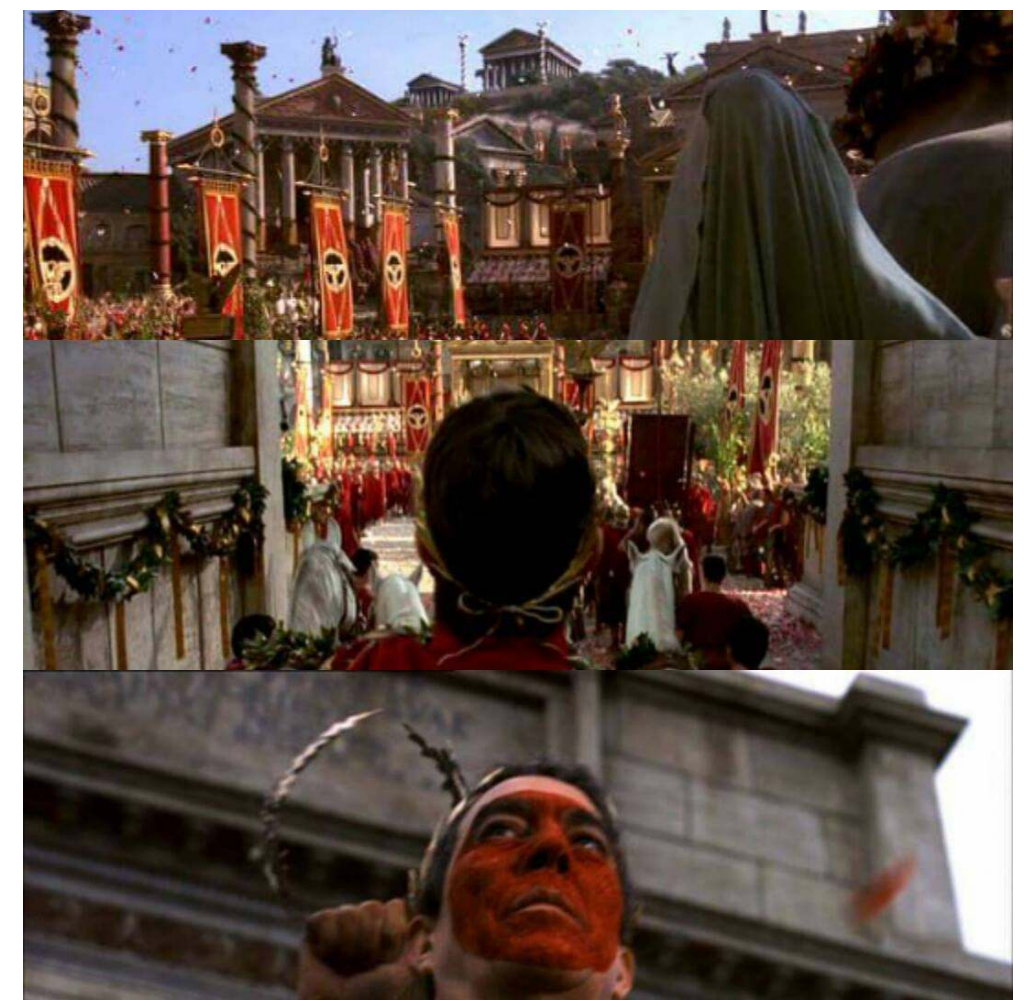

Fig. 20
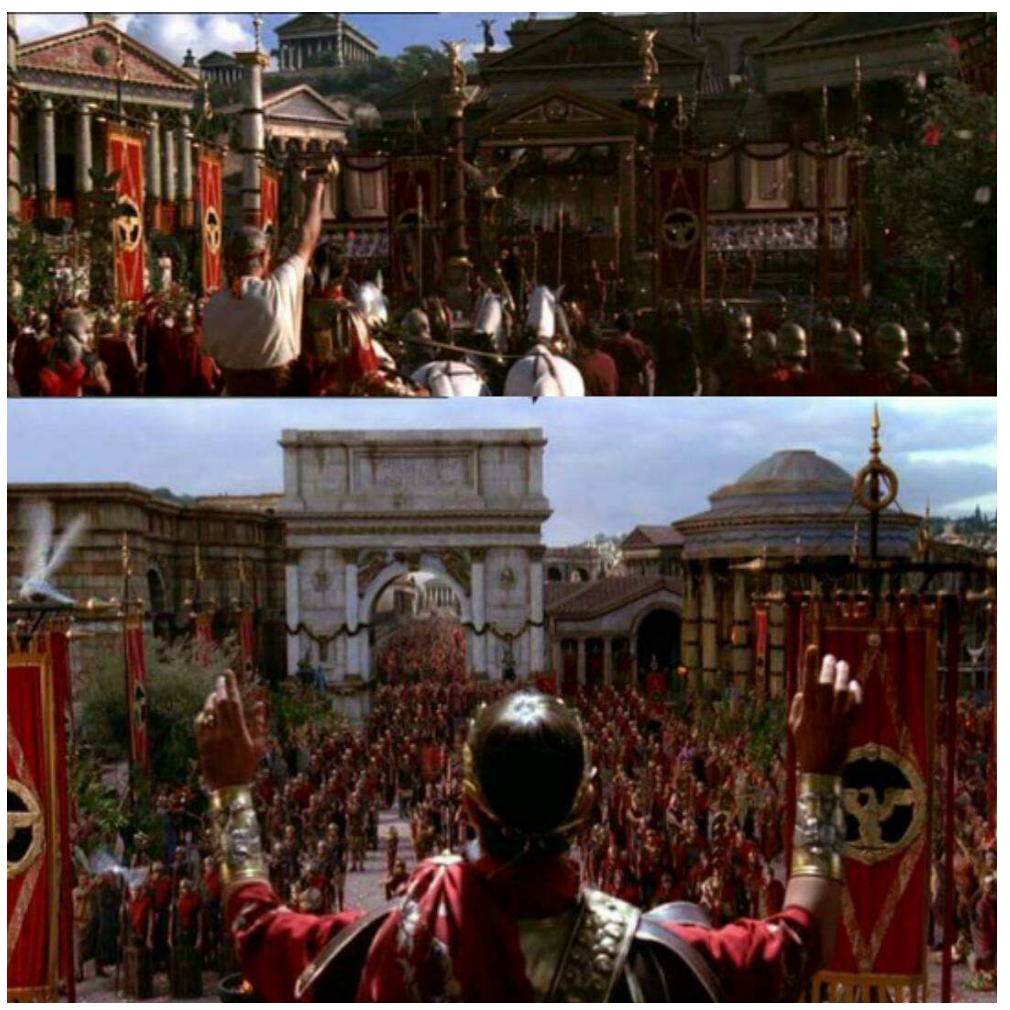

Fig. 21 


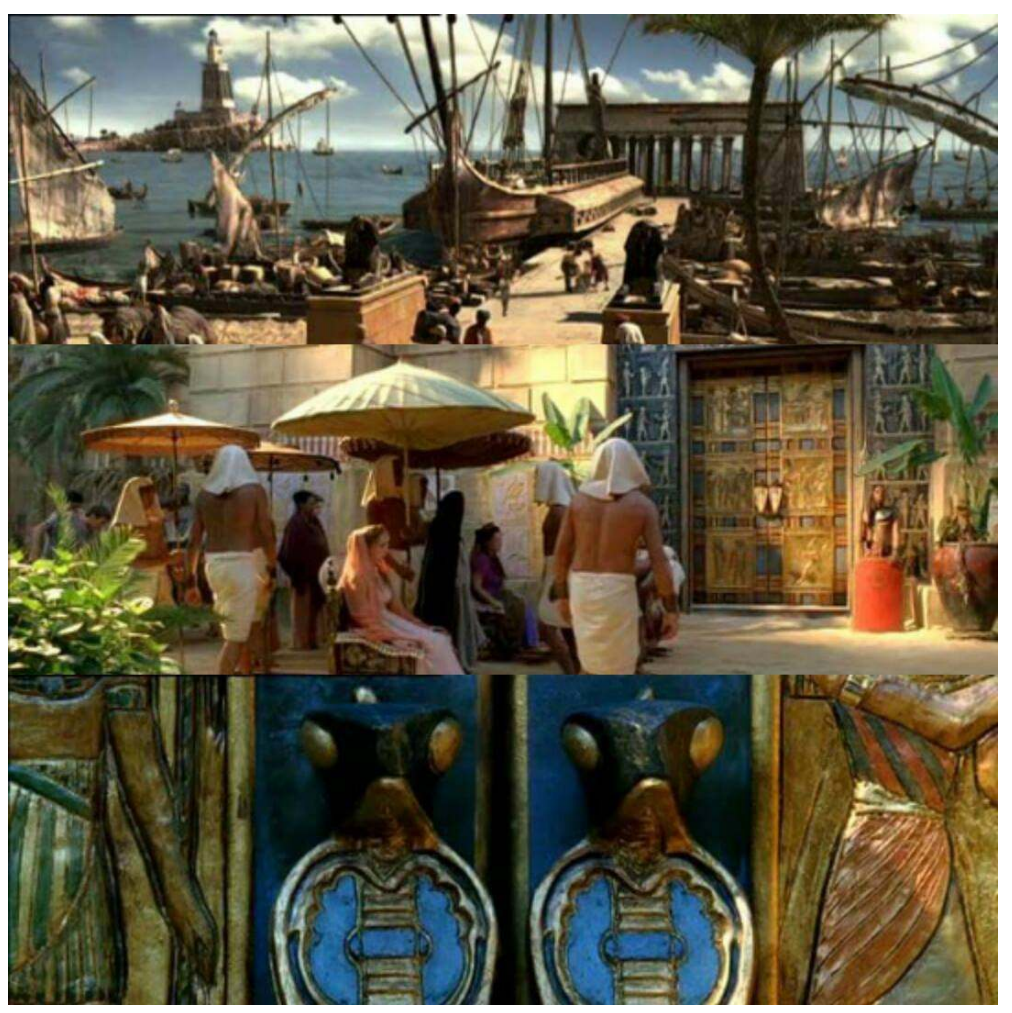

Fig. 22

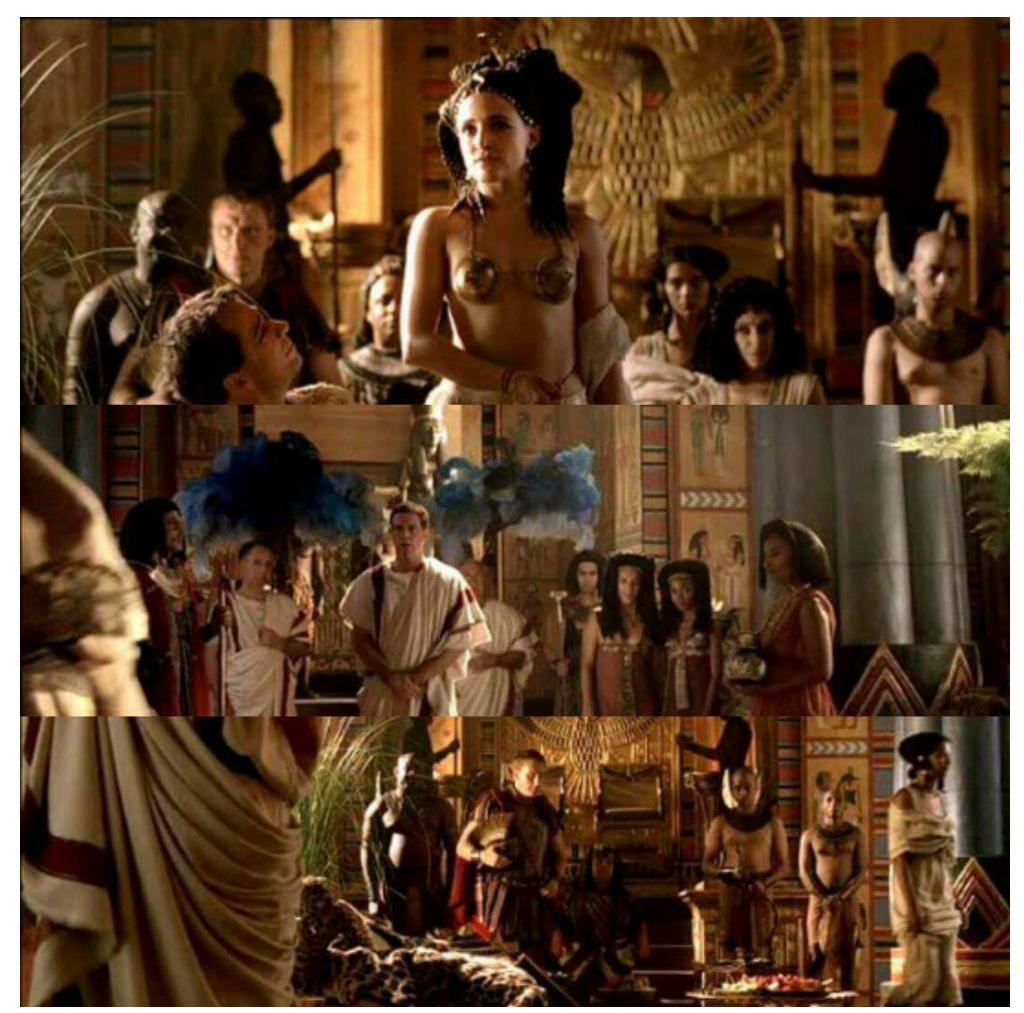

Fig. 23

29 Ainsi, Rome dans Rome, du fait de la sérialité qui conditionne une narration qui se déploie ou s'accélère dans le temps, transpose et métaphorise sur le plan spatial et paysager les singularités génériques de la série : elle orchestre sur le plan spatial et paysager le tempo 
de la série qui recrée une dynamique historique autre. Par la dilatation ou le resserrement des plans intérieur/extérieur, c'est le temps de l'histoire antique qui se trouve alors entièrement redessiné selon des logiques ornementales et paysagères : Rome nous donne à lire une version autre de l'histoire de Rome. De même, en reprenant une partie des stéréotypes de représentation - nourris du vraisemblable historique du fait de l'importance accordée aux realia antiques - la Rome de Rome, présente des caractéristiques similaires aux villes-monde antiques de nos univers visuels contemporains à tel point que l'on peut conclure à son propos :

Au travers de la figure urbaine de Rome, figure tentaculaire et modèle urbain qui se répète à l'intérieur du jeu et entre les jeux [tout comme elle se répète à l'intérieur de l'épisode et entre les épisodes], c'est un réseau d'images de paysage urbain qui se déploie et dans lequel notre imaginaire contemporain peut plonger à satiété. [...] Rome, tout comme Athènes, en tant que ville-paysage marqueur de l'Antiquité, étendent leur grammaire iconographique à l'ensemble de nos modalités de perception et de réception de la société à laquelle elles appartiennent originellement. Ce faisant, elles deviennent, par l'abolition des frontières spatio-temporelles qu'offre l'univers [visuel], des villes-mondes exploitables par l'infini des possibles ${ }^{45}$.

\section{L'ornement-paysage}

31 Si l'alternance paysage/ornement rythme la série et permet au spectateur de s'immerger dans le monde romain antique, il se produit aussi un phénomène intéressant sur le plan de l'appréhension du paysage: placé sur le même plan que les scènes intérieures qui mettent à l'honneur les ornements picturaux ou la décoration intérieure, le paysage, pour rester lisible en tant que tel et faire sens sans trop d'anachronisme (c'est pourtant le paysage actuel de l'Italie qui est à l'écran), doit être lu et analysé par le spectateur exactement comme les éléments ornementaux qui reproduisent à l'identique des realia archéologiques issus des productions artistiques romaines. Ainsi, ces reproductions qui reprennent les objets et les œuvres de l'art romain canonique offre leur cadre esthétique et conceptuel au paysage naturel : la série Rome porte donc à son comble la mise à distance du schème panoramique au profit de celui de l'ornement pour apprécier le paysage. Sur le plan de la mise à l'écran et de l'enchaînement des plans et des séquences, on pensera ici, par exemple, à l'épisode 3 de la saison 1 où l'alternance des scènes paysages extérieur/ornement intérieur participe de la tension dramatique : le tempo s'accélère au fur et à mesure que la situation politique s'envenime pour les protagonistes : l'épisode s'ouvre sur une scène haute en couleur à nos yeux où la domina, Atia, fouette son esclave préféré, Castor, pour passer ses nerfs car César vient de franchir le Rubicon et elle craint les conséquences pour l'ensemble de la familia des Julii. La scène se passe dans un coin de l'atrium décoré des masques ou de portraits en buste représentant le visage des ancêtres de la Gens Julia, les imagines. Les couleurs vives de la pièce - le rouge, contraste avec les masques sombres placés dans de petites niches dont le contour est rehaussé d'ornements en guirlandes florales dorées [fig.24]. 


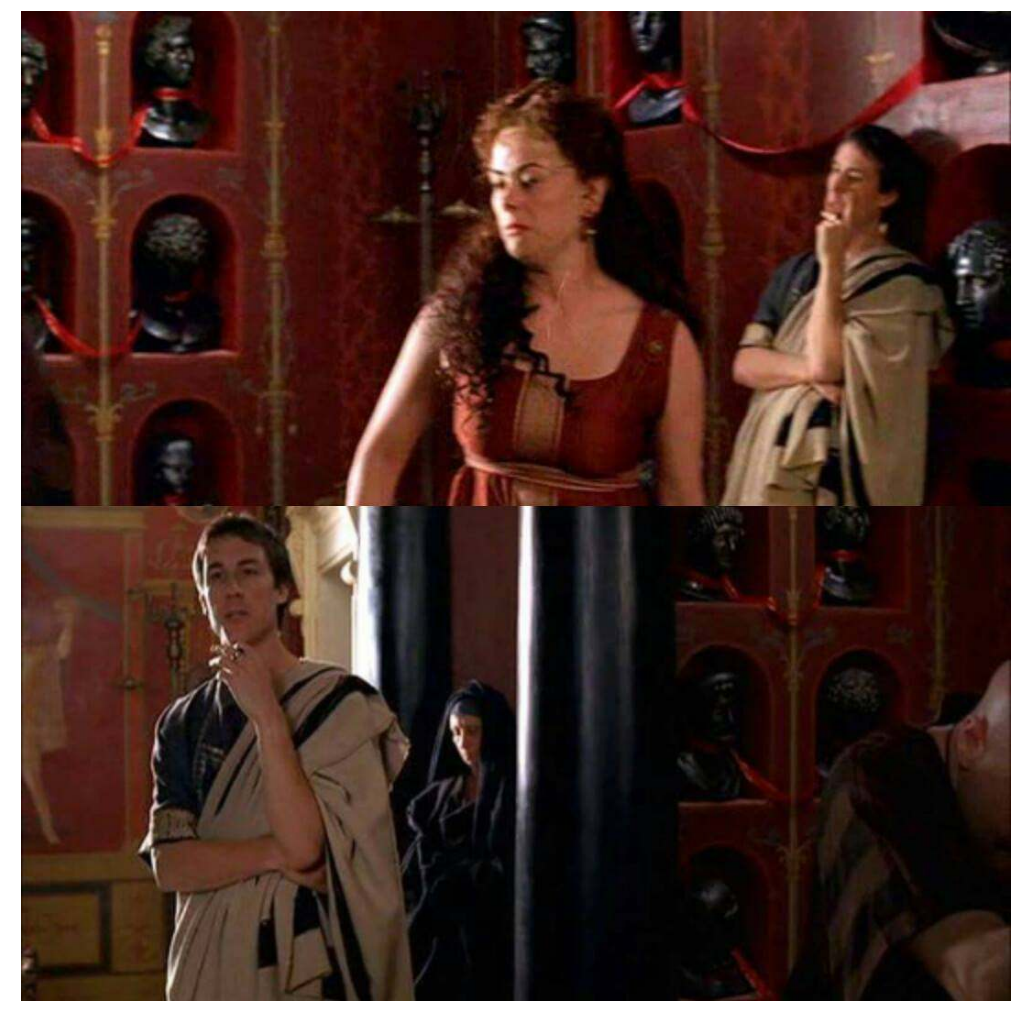

Fig. 24

32 Cette décoration travaillée rappelle les ornements complexes du style pictural impérial. Le plan s'enchaîne avec une discussion entre Pompée et Caton dans le très bel atrium végétal de Pompée dont le jardin intérieur luxuriant est orné de statues comme les 
peintures pompéiennes, dont celle de la Maison du bracelet d'or, par exemple, en témoigne [fig.25].

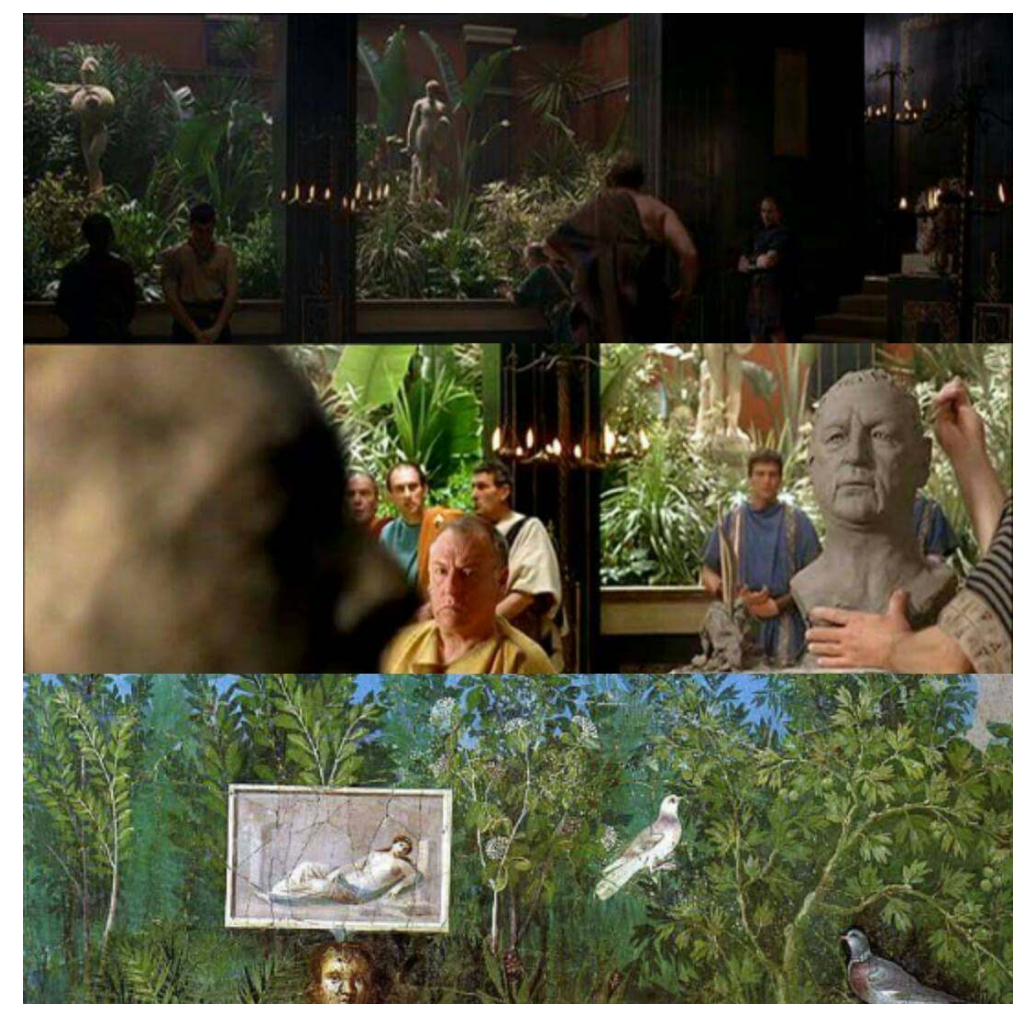

Fig. 25

Enfin, un cut nous projette en rase campagne latine, dans un paysage naturel, où les armées de César se hâtent de regagner les abords de l'Urbs. Une même logique s'observe pour la suite de l'épisode où la séquence en paysage naturel est coupée pour montrer ensuite l'intérieur, cette fois plus modeste, de la maison de Vorenus, soldat de César, où sa femme l'attend, en prière, au pied du lararium: la décoration humble témoignant de leur niveau social de plébéiens n'en est pas moins ornée, la peinture de l'autel aux dieux Lares reproduisant à l'identique celle du lararium de la Maison des Vettii [fig.26]. 


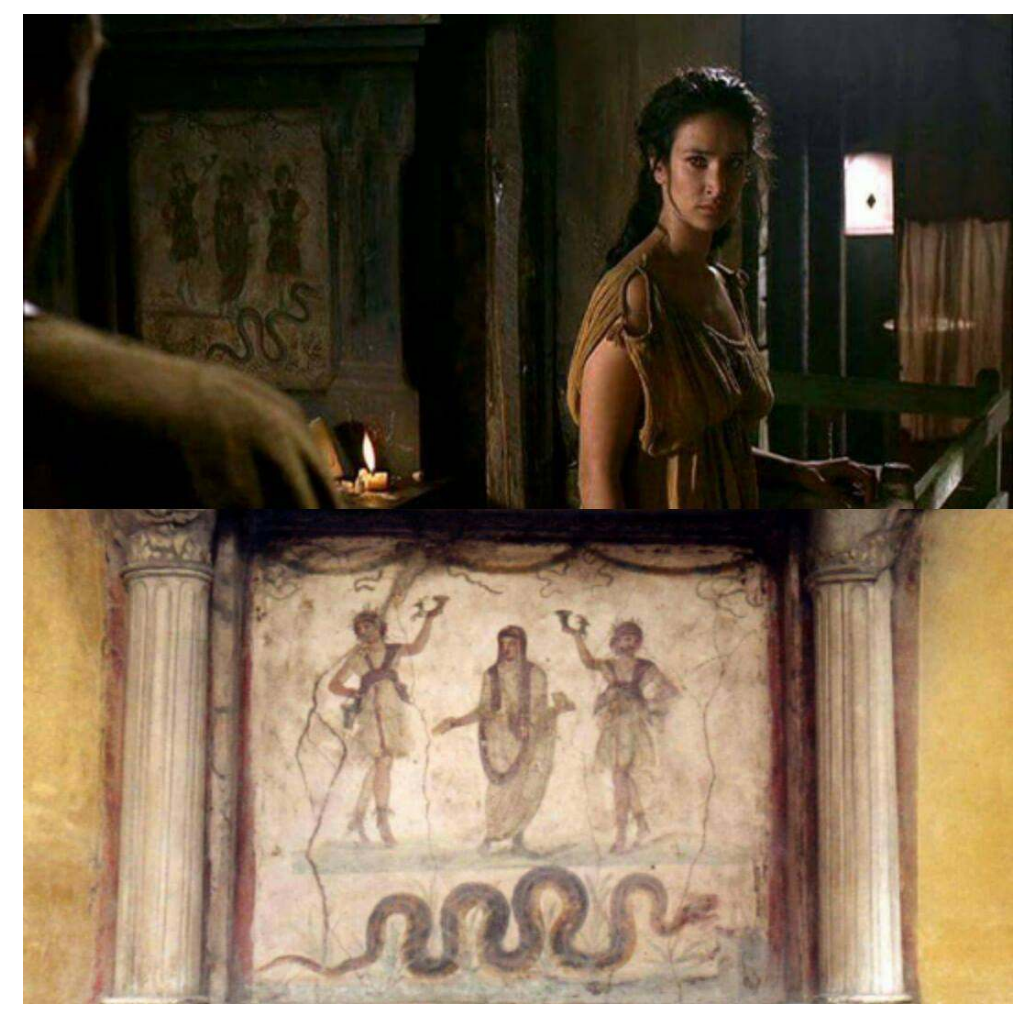

Fig. 26 le tout en seulement 5 minutes. À chaque cut ou enchaînement d'une séquence à l'autre, s'opère un lien fort entre les images : on passe de l'ornement au paysage et du paysage à l'ornement. Lors de la scène d'ouverture de l'épisode, c'est le mouvement de caméra qui fait glisser l'œil de droite à gauche, focalisant sur un détail de l'ornement pictural (les guirlandes dorées), saisissant les protagonistes dans leur action (Aitia fouettant Castor, Brutus prononçant «César a franchi le Rubicon » au moment même où il se déplace dans la pièce et où la caméra bouge pour le suivre) pour s'arrêter de nouveau sur un détail de l'ornement pictural (les mêmes guirlandes à l'autre bout du mur) pour enchaîner sur l'autre séquence, introduite par la marche dynamique de Caton qui guide l'œil jusqu'au jardin de l'atrium où Pompée médite, pour ensuite focaliser plein cadre sur les deux hommes politiques filmés tantôt de face avec pour fond la décoration des murs de l'atrium ou de dos, avec pour fond le jardin orné de nombreuses sculptures. De nouveau c'est l'ornement - et ici l'ornement végétalisé avec les statues dans le jardin - qui permet de glisser vers le paysage naturel à l'écran. Le plan large avec les soldats au galop se resserre ensuite sur l'intérieur de chez Vorenus en filmant les mains de Niobé (sa femme) qui coupe des légumes, puis remonte sur son visage en la filmant plein cadre avec pour fond, la peinture du lararium, elle-même représentant un paysage stylisé sacré avec le serpent Agathodémon associé à la fertilité de la terre [fig. 24 à 26]. C'est donc bien entre ornement et paysage que l'enchaînement des séquences et des plans se joue et que se tisse le tempo alerte de cet épisode. Ce jeu d'alternance rapide se retrouve tout au long de l'épisode mais aussi tout au long de la série.

ll semblerait alors que Rome, tout en inventant une ligne de réception singulière par la lecture ornementale du paysage, instaure un régime de perception qui serait propre à notre regard de contemporain car nous retrouvons, quelques années plus tard, dans 
l'univers vidéoludique et du cinéma d'animation, une approche qui, sur le plan théorique, mobilise bien plus l'ornemental que le panoramique pour saisir la dimension paysage de l'Antiquité à l'écran. C'est ce que nous avons noté à propos du jeu Apothéon (2015) et de l'animé Hercule (1997) :

Ainsi, le statut de ces divers ornements qui réferent tous à l'univers visuel et artistique de la Grèce antique, essentiellement archaïque et classique, entretient une ambiguité générique tant sur le plan de la nature de l'espace perçu par le joueur que sur celle du référent des objets représentés et de l'espace vidéoludique : que regardons nous lorsque nous voyons, dans le jeu, lors d'une même étape, une frise avec des vagues qui délimitent un registre supérieur d'un registre inférieur, placée sur le même plan qu'un élément rocheux, un arbre ou une route du paysage dans lequel évolue l'avatar, comme dans l'épisode de la forêt d'Artémis? Regardons-nous un détail du vase qui contient la scène ou sommes-nous dans le paysage qui sert d'écrin à l'action? Mieux encore, au sein de cette confusion, quelle place attribuer à la reproduction d'un élément de décor, d'un ornement ou d'une grande cuvre artistique « réelle » faisant partie du répertoire mondial artistiqu ${ }^{46}$ ?

$\mathrm{Si}$, à l'écran, la série Rome, du fait de sa teneur historique, ne va pas aussi loin que le travail plastique vidéoludique d'Apotheon, le principe de sérialité œuvrant à la mise sur le même plan du paysage et de l'ornement travaille dans un sens identique. C'est, de notre point de vue, une très grande singularité visuelle du paysage dans cette série antiquisante.

\section{Paysage antique, sérialité, série éternelle}

Ainsi, le paysage dans les séries à thème antique, oscillant entre d'un côté, l'insertion de la 3D visant à hypertrophier le paysage naturel de façon à l'aligner sur l'esthétique de la "maximisation" héritée de l'univers vidéoludique ${ }^{47}$ (Atlantis) et de l'autre, la confrontation, voire la marginalisation de la 3D (Odysseus) semble instaurer, par rapport à l'univers vidéoludique et aux décors cinématographiques du péplum de la même génération dont il s'inspire, un retour au "naturel». Mais un "naturel» mâtiné de virtuel. Ou, pour le dire autrement, une «virtualité raisonnée» favorisant ainsi l'immersion historique du spectateur dans un univers qui pourtant traite de sujet mythologique et merveilleux. Avec la série Rome, novatrice en la matière, on assiste à un phénomène paradoxal : cette série, qui s'est voulue pourtant très proche des réalités historiques et anthropologiques de la Rome tardo-républicaine et impériale, opère par le biais du principe de sérialité une remise en question du régime de lecture plastique du paysage. Congédiant, du fait de la mise sur le même plan esthétique de l'ornement et du paysage, le schème du panoramique, cette série qui articule étroitement tempo narratif sériel et récit historique antique, redessine les contours spatio-temporel de l'objet " antiquité ", de sorte que, Rome traitant de la Rome intemporelle, devient elle-même série éternelle. Cette perspective se laisse déjà appréhender dès le générique qui ouvre chacun des épisodes et qui déroule, tel un papyrus contemporain, en animation surimposée sur des plans cinématographiques, des éléments archéologiques réels synthétisant en une vue dynamique tel un tissu brodé et orné, les grands symboles religieux et culturels, les grands éléments du quotidien de la vie des romains. En travaillant plastiquement l'Antiquité, même au prix d'anachronismes, de simplifications ou d'erreurs historiques ${ }^{48}$, Rome fait sortir ce « quelque chose de l'Antiquité qui dialogue encore avec notre culture de contemporains ", elle met en scène ce qui peut encore faire sens, ce qui est la partie de 
l'Antiquité « mal imprimée » mais « suffisante » pour que tous puissent encore y trouver quelque intérêt.

Ainsi nous avons pu, nous semble-t-il, mieux apprécier comment la série, en tant que genre, réinvente un mode de représentation du paysage, non plus compris selon la définition à dominante panoramique, issue du champ de l'Esthétique, mais en invitant à rejouer le débat sur l'ornement. C'est entre l'ornement et le panorama que la série déplace les enjeux de la représentation du paysage à l'écran comme en témoigne le cas spécifique du paysage antique.

\section{Bibliographie}

André (Laury-Nuria) \& Lécole-Solnychkine (Laury-Nuria), «Paysages et topoï dans le péplum grec contemporain: de l'esthétique de la ruine à sa résurrection virtuelle », Anabases, 18, 2013, p. 109-131.

André (Laury-Nuria) \& Lécole-Solnychkine (Laury-Nuria), « L'Antiquité vidéoludique, une résurrection virtuelle? », Nouvelle Revue d'Esthétique, PUF, n¹1, 2013, p. 87-98.

André (Laury-Nuria), Game of Rome. L'Antiquité vidéoludique, Caen, Passage(s), coll. Essais, 2016.

André (Laury-Nuria), De l'île au texte. Le paysage-palimpseste des anciens Grecs (IIIe av. J.-C - Ve ap. J.-C.), Paris, Les Belles Lettres, à paraître.

4 André (Laury-Nuria), «Entre histoire et géographie, écrire le paysage chez Diodore de Sicile et Strabon. Le cas des îles occidentales ", in Orient \& Méditerranée 19, 2016, à paraître.

Auerbach (Erich), Mimésis. La représentation de la réalité dans la littérature occidentale, Paris, Gallimard, Coll. Tel, 1977.

Barbet (Alix), La Peinture murale romaine. Les styles décoratifs, Paris, Picard, 2009 (2e édition).

Berque (Augustin), Les Raisons du paysage. De la Chine antique aux environnements de synthèse, Paris, Hazan, 1995. -- (dir.), Cinq propositions pour une théorie du paysage, Seyssel, Éditions ChampVallon, Collection Pays/Paysage, 1994.

-- et Besse (Jean-Marc) et alii (éds.), Les Enjeux du Paysage, Recueil n8, Bruxelles, OUSIA, 1997.

-- et Conan (Michel), Donadieu (Pierre).

Besse (Jean-Marc), Voir la terre, six essais sur le paysage et la géographie, Paris, Actes Sud/ ENSP/Centre du paysage, 2000.

Bessières (Vivien), « Rome : un péplum enfin réaliste ? », TV/Series [En ligne], 1 | 2012, mis en ligne le 15 mai 2012, consulté le 03 août 2016. URL : http://tvseries.revues.org/1066.

Bigg (Charlotte), « The Panorama or la Nature à coup d'oeil » in Erna Fiorentini, Observing Nature - Representing Experience. The Osmotic Dynamics of Romanticism 1800 - 1850, Berlin, Dietrich Reimer Verlag GmbH, 2007.

3 Camporesi (Piero), Les Belles contrées, Paris, Le Promeneur, 1995.

Cauquelin (Anne), L'Invention du paysage, Paris, PUF, 2000. Ritter (Joachim), Paysage, Fonction de l'esthétique dans la société moderne, Besançon, Les Éditions de l'Imprimeur, 1997. 
Actes des journées d'étude des 20 et 21 septembre 2010, Bibliothèque de l'Université de Paris 8 , 2010.

67 Schefold (Karl), "Origins of Roman Landscape Painting », The Art Bulletin, Vol.42, No.2, 1960, p.87-96.

68

Siebert (Gérard), Nature et paysage dans la pensée et l'environnement des civilisations antiques, Paris, de Boccard, 1996.

69 -- «Imaginaire et images de la grotte dans la Grèce archaïque et classique », in Gérard Siebert (éd.), Nature et paysage dans la pensée et l'environnement des civilisations antiques, Paris, De Boccard, 1996, p.47-57. Métamorphoses, IV, 6 », Rev. de Philologie, LXXIII, 1999, vol.2, p.257-277.

73 -- Loca Horrida. L'espace des animaux sauvages dans le monde romain entre la fin de la République et le Haut Empire, thèse de doctorat sous la direction d'Agnès Rouveret, Paris $\mathrm{X}$ Nanterre, 2004. 


\section{NOTES}

1. Voir, entre autres, Berque (Augustin), Les Raisons du paysage. De la Chine antique aux environnements de synthèse, Paris, Hazan, 1995. -- (dir.), Cinq propositions pour une théorie du paysage , Seyssel, Éditions ChampVallon, Collection Pays/Paysage, 1994. -- et Besse (Jean-Marc) et alii (éds.), Les Enjeux du Paysage, Recueil n8, Bruxelles, OUSIA, 1997. -- et Conan (Michel), Donadieu (Pierre), Lassus (Bernard), Roger (Alain), Mouvance, cinquante mots pour le paysage, Paris, Éditions de la Villette, 1999. Besse (Jean-Marc), Voir la terre, six essais sur le paysage et la géographie, Paris, Actes Sud/ ENSP/Centre du paysage, 2000. Camporesi (Piero), Les Belles contrées, Paris, Le Promeneur, 1995. Cauquelin (Anne), L'Invention du paysage, Paris, PUF, 2000. Ritter (Joachim), Paysage, Fonction de l'esthétique dans la société moderne, Besançon, Les Éditions de l'Imprimeur, 1997. Roger (Alain), Court traité du paysage, Paris, Gallimard, 2008 (1997).

2. Entre autres, Camporesi (Piero), Les Belles contrées, Paris, Le Promeneur, 1995, Le Dantec (JeanPierre), Jardins et paysages. Une anthologie, Paris, éditions de la Vilette, 1997 et Michael Jakob, Le Paysage, Paris, Infolio, 2008.

3. Entre autres, Rouveret (Agnès), « Pictos ediscere mundos. Perception et imaginaire du paysage dans la peinture hellénistique et romaine », Ktema, 29, 2004, p.325-344. -- "Les paysages de Philostrate ", in Michel Costantini, Françoise Graziani et Stéfane Rolet (éd.), Le défi de l'art. Philostrate, Callistrate et l'image sophistique, Rennes, La Licorne, 2006, p.63-76. Saliou (Catherine) et alii, Les sources de l'histoire du paysage urbain d'Antioche sur l'Oronte. Actes des journées d'étude des 20 et 21 septembre 2010, Bibliothèque de l'Université de Paris 8, 2010. Schefold (Karl), «Origins of Roman Landscape Painting », The Art Bulletin, Vol.42, No.2, 1960, p.87-96. Siebert (Gérard), Nature et paysage dans la pensée et l'environnement des civilisations antiques, Paris, de Boccard, 1996. -« Imaginaire et images de la grotte dans la Grèce archaïque et classique », in Gérard Siebert (éd.), Nature et paysage dans la pensée et l'environnement des civilisations antiques, Paris, De Boccard, 1996, p.47-57. Spencer (Diana), Roman Landscape : Culture and Identity, Cambridge University Press, 2010. Thompson (Dorothy B.), «The garden of Hephaïstos » in Hesperia VI, 1937, p.396-425. Trinquier (Jean), « le motif du repaire des brigands et le topos du locus horridus : Apulée, Métamorphoses, IV, 6 ", Rev. de Philologie, LXXIII, 1999, vol.2, p.257-277. -- Loca Horrida. L'espace des animaux sauvages dans le monde romain entre la fin de la République et le Haut Empire, thèse de doctorat sous la direction d'Agnès Rouveret, Paris X Nanterre, 2004. Ainsi que l'ensemble de nos travaux.

4. Voir nos travaux en Sciences de l'Antiquité, dont la publication de notre thèse de doctorat. André (Laury-Nuria), De l'île au texte. Le paysage-palimpseste des anciens Grecs (IIIe av. J.-C - Ve ap. J.C.), Paris, Les Belles Lettres, à paraître.

5. « De l'Antiquité, il sera question tout au long de notre réflexion. Elle est la figure du désir et de l'absence, elle est l'aimé(e) désiré(e), malmené(e), nié(e) puis révélé(e), (re)trouvé(e) et partant, réinventé(e) selon la célèbre formule rimbaldienne l'«amour est à réinventer ». L'Antiquité se définit précisément par l'absence. L'absence est sa nature fondamentale - d'où les figures qui ont eu la charge de la signifier, comme la ruine. L'Antiquité c'est l'essence de l'absence. Elle ne cesse de se définir par sa ruine, son abandon, son oubli, sa mise à l'écart, sa mort et ce, jusque dans nos débats les plus actuels». André (Laury-Nuria), Game of Rome. L'Antiquité vidéoludique, Caen, Passage(s), coll. Essais, 2016, p.10. Enfin, «matière antique ", nous entendons la somme de tous les objets, usages, concepts et imaginaires qui constituent la civilisation antique. Le terme même de «matière " engage une plasticité de ces objets, qui ouvre à la possibilité de leur "mise en récit » ou de leur «mise en fiction »: nous parlons alors de «fictions archéologiques », et/ou de 
topoïétique (Marc Ferniot): il s'agit de singulariser le lieu commun (une culture que nous fantasmons comme le mythe de nos origines) par l'entremise de la fiction.

6. «Ce processus de réception de l'Antiquité appartient en propre à notre regard de contemporains. Et, ce qui le distingue du long cheminement des diverses modalités de réception à travers les siècles et les cultures qui nous précèdent, c'est qu'il transforme l'Antiquité, du fait même de la nature du médium qui le constitue, pour en donner une version autre. C'est donc en terme de copie et d'altérité - mais d'altérité duelle, à la fois même et autre, proche et lointaine, semblable et dissemblable du modèle [...] que nous penserons ce processus de réception contemporaine de l'Antiquité en tentant de montrer en quoi, justement, il sort des ornières de la mimêsis. ", André (Laury-Nuria), Game of Rome. L'Antiquité vidéoludique, op.cit., p.11.

7. «Quelle est donc la modalité de présence de l'Antiquité dans l'image, qui se tisse, au-delà ou en parallèle de la question de la mimêsis et qui fait de l'image vidéoludique antiquisante une copie qui simultanément ressemble et ne ressemble pas à sa matrice antique, une copie qui reproduit une partie de l'Antiquité mais pas l'ensemble des référents (motifs, paysage, ornements, architecture etc.), trace incomplète, silhouette, résultat partiel d'un tampon qui n'aurait pas imprimé l'intégralité du motif comme si l'on n'avait pas exercé la même pression partout et qui, pourtant, se donne clairement comme telle pour le joueur ? Trace, silhouette, impression... ces déclinaisons de formes appartiennent, nous semble-t-il, au registre de l'empreinte[...] Avec le changement médiumnique, c'est un changement esthétique qui s'opère et avec lui, une redéfinition de l'art antique, qui, pour une part, s'opère selon un réalignement du paradigme antique sur des critères esthétiques qui, pour servir pleinement de composante à la nature de l'image virtuelle actuelle, n'en constituent pas moins des éléments esthétiques à l'histoire au long cours ». André (Laury-Nuria), Game of Rome. L'Antiquité vidéoludique, op.cit., p. 13-14.

8. Voir, entre autres, André (Laury-Nuria) \& Lécole-Solnychkine (Sophie), « Paysages et topoï dans le péplum grec contemporain: de l'esthétique de la ruine à sa résurrection virtuelle ", Anabases, 18, 2013, p. 109-131.

9. Voir André (Laury-Nuria) \& Lécole-Solnychkine (Sophie), "L'Antiquité vidéoludique, une résurrection virtuelle? ", Nouvelle Revue d'Esthétique, PUF, n¹1, 2013, p. 87-98.

10. Nous avons pu le vérifier par l'analyse de plusieurs jeux vidéos, voir André (Laury-Nuria), Game of Rome. L'Antiquité vidéoludique, op.cit. Cette esthétique de la dilatation et des panoramas paysagers grandioses se retrouve dans plusieurs péplums de la même génération 2010's : 300 de Zack Snyder, Legendary Pictures, 2007 ; Le Choc des Titans de Louis Leterrier, Warner Bros, 2010 ; Les Immortels de Tarsem Singh, Universal Pictures, 2011; la Colère des Titans de Jonathan Liebesman, Warner Bros, 2012; 300 : La Naissance d'un empire de Noam Murro, Warner Bros. Pictures, 2014 ; Hercule de Brett Ratner, Paramount Pictures, 2014 ; Pompéi de Paul W.S. Anderson, Constantin Film, 2014 ; Noé de Darren Aronofsky, Disruption Entertainment, 2014 ; L'Exode : Dieux et Rois de Ridley Scott, Scott Free Productions, 2014 et Les Dieux d'Égypte de Mystery Clock Cinema, 2016.

11. Rome, série télévisée américano-britannico-italienne, 22 épisodes, 2 saisons, John Milius, William J. MacDonald et Bruno Heller, HBO et BBC 2, 2005-2007.

12. Atlantis, série télévisée britannique, 26 épisodes, 2 saisons, Johnny Capps, Julian Murphy et Howard Overman, 2013-2015.

13. Odysseus, série télévisée péplum franco-italo-portugaise, 12 épisodes de 45 minutes, 1 seule saison, Frédéric Azémar et Stéphane Giusti, ARTE, 2013.

14. Voir, entre autres, Mérot (Alain), Du paysage en peinture dans l'Occident moderne, Paris, Gallimard, 2009.

15. Pour donner un ordre d'idée : le budget final de la série s'élève à 14 millions d'euros, un budget restreint qui équivaut au «budget du pilote de la série Rome» selon Frédéric Azémar, Sylvain Merle, «Odysseus revisite le mythe d'Ulysse » [archive], Le Parisien, 13 juin 2013, article consulté le 29/07/2016. 
16. Rappelons quelques données théoriques et historiques : «First patented in the UK by the Irish painter Robert Barker in 1787, the panorama is a large circular painting offering the spectator a 360 degree view of a landscape, but the term also describes the purpose-built structure in which it is housed. The word is a neologism from the Greek meaning "an all-embracing view," or as Barker first described his set-up, "la nature a coup d'oeil." To give viewers the impression they were surrounded by an actual landscape, Barker conceived a circular room whose walls are entirely covered by a continuous, painted canvas lit from above. The spectators entered the room using an underground passage suitably darkened to enhance the effect when they emerged onto a central island from which they could view the scene. A number of contrivances are listed in the patent, all intended to enhance the illusion. Important was to suppress any external referent against which the panorama could be compared, to meticulously render adequate proportions and perspective on the curved surface, to convey realistic illumination and shadows, to compensate for the sagging of the painting under its weight and to hide the canvas' junction lines. ", Bigg (Charlotte), « The Panorama or la Nature à coup d'oeil » in Erna Fiorentini, Observing Nature - Representing Experience. The Osmotic Dynamics of Romanticism 1800 - 1850, Berlin, Dietrich Reimer Verlag GmbH, 2007, p. 73.

17. Cette proximité, cette intimité créée par la virtualité raisonnée du paysage antique reconstruit nous paraît être un facteur non négligeable travaillant dans le sens de l' "effetretour ", notion que nous interrogeons comme marqueur et indicateur du travail de notre regard de contemporain, comme conséquence du processus de réception de la matière antique. Voir, entre autres, André (Laury-Nuria), Game of Rome, op.cit., p. 85-102.

18. «Il s'agit de questionner le processus poïtique dont relèvent les décors paysagers du jeu. Nous verrons en quoi ce processus s'apparente, dans les cas étudiés, à la mise en œuvre d'une interprétation construite de l'Antiquité. [...] Pour construire cette image stéréotypée à l'origine du «syndrome Acropole», le game designer collecte des éléments architecturaux et paysagers dont la véracité historique est attestée afin de constituer un catalogue définissant l'univers de référence du projet. [...] Cependant, une dominante de perception se dégage de l'ensemble des travaux réalisés quelque soit le game designer [...] L'amplification régit en effet la représentation de la ville de Rome dans les trailers de CivCity Rome, Caesar IV, Ryse : Son of Rome etc., elle concerne aussi la ville de Carthage dans Rome Total War II et l'ensemble des infrastructures urbanistiques et palatiales dans God of War, Shadow of the Colossus ou encore Rise of the Argonauts pour ne citer qu'eux. Le procédé de maximisation qui contribue à la spectacularisation des paysages vidéoludiques antiques tend à réduire l'effet de réel et le référent historique et archéologique sur lequel repose pourtant le procédé de stéréotypie. C'est dans cette tension, créée par ce procédé qui part d'éléments réels pour les désolidariser de leur contexte historique et qui les fictionne ou les "déréalise " par amplification de leurs caractéristiques visuelles, que réside le mouvement chiasmatique définissant l'esthétique anadyomène de l'image contemporaine de l'Antiquité ». André (Laury-Nuria), Game of Rome, op.cit., p. 69 et p. 72. Notons que ce phénomène du "syndrome Acropole» reposant sur l'esthétique de la maximisation paysagère se retrouve exploité dans les péplums hollywoodiens de la même génération : voir le corpus mentionné dans la note $10 \mathrm{du}$ présent article.

19. «Selon quelles modalités s'effectue cette rencontre entre les références historiques et l'univers de fiction dans lequel le joueur s'immerge? Répondre à cette question implique la découverte du phénomène de réception - qui dépend d'un processus esthétique - tout à fait singulier de la matière antique que proposent les jeux vidéo. Nous l'avons dit, au travers de la mise en image vidéoludique de l'Antiquité, se joue un processus de réception qui ne fonctionne qu'à partir d'une infime partie de l'objet, une petite partie de la matière à représenter: l'Antiquité grecque ou romaine. Cette partie de la matière antique, mal imprimée, est comme une empreinte partielle mais suffisante pour être signifiante aux yeux du joueur. Si elle reste signifiante à l'écran, c'est parce qu'elle relève d'une dimension stéréotypée: des critères a minima 
fonctionnent comme marqueurs identitaires à l'écran, selon une logique métonymique. Ainsi en va-t-il du Colisée pour Rome - et par extension pour toute l'Antiquité romaine - du Parthénon juché sur l'Acropole pour Athènes - et toute l'Antiquité grecque - du Portique Ouest du site Cnossos (celui-là même dont Arthur Evans avait déjà proposé une anastylose fort décriée en son temps) pour la civilisation minoenne, de la porte d'Ishtar pour Babylone et parfois même Troie, du Phare d'Alexandrie ou des Pyramides pour l'Égypte pharaonique ou hellénistique etc. Il en va de même pour les personnalités historiques qui arpentent l'univers vidéoludique : une logique d'antonomase se met en place et voilà qu'Homère devient le symbole de la Grèce antique, que César, Néron etc. - avec des «erreurs» historiques de taille - deviennent les parangons de l'Empire romain à son apogée, que Pan, Atalante, Héraclès, Jason et bien d'autres héros ou divinités antiques se font herméneutes et guides de l'avatar au sein du jeu et transposent à chacune des étapes - tels les Pénates de Troie ramenés et transportés par Énée en terre étrangère - toute l'Antiquité dont ils peuvent être chargés. Ce phénomène, nous l'avons appelé le " syndrome Acropole », André (Laury-Nuria), Game of Rome, op.cit., p. 66-67.

20. On observe le même jeu de va-et-vient entre le centre d'Ithaque symbolisé par le palais de Pénélope et Télémaque et l'arrière-pays, la chôra d'Ithaque, dans la série Odysseus.

21. Nous y venons dans la seconde sous-partie du présent développement.

22. «Pour se représenter le monde, la pensée grecque - qu'elle se manifeste dans les arts visuels et plastiques ou dans la littérature - a eu recours à des objets métaphoriques dont le rapport d'échelle permettait de mettre à portée de main le monde habité, de le voir, à échelle réduite. Ce faisant, ces objets sont passés au stade de modélisateurs engageant un jeu de transferts entre forme et création [...] Or, si le paysage insulaire a prêté sa forme au paysage du monde entier, l'île entendue comme paysage paradigmatique du monde connu par les Anciens a connu une fortune inégale et surtout, de profondes mutations tant formelles que fonctionnelles. On distingue en effet quatre grandes étapes dans l'instrumentalisation de l'île comme paysage informant la cosmographie antique. [...] Un premier moment se dessine dès la poésie archaïque d'Homère, où l'île est la figure du monde dans la pensée épique. [...] L'image de l'île est faite de cercles concentriques : le cercle des rivages côtiers, celui de la plaine qui s'étend vers le centre de l'île, entre la bande de sable et le piémont et enfin le centre de l'île constitué par la montagne qui s'élève et domine en structurant, par sa percée axiale, l'espace insulaire et clôt sur lui-même qu'est l'île. Cette composition à la fois géographique, physique et archétypale, stylisée et stéréotypée, correspond à l'organisation matérielle et plastique du bouclier : il est bombé au centre et il étend sa peau de bœuf jusqu'à la bordure de bronze qui l'encercle et le solidifie tout en l'ornant d'une dimension esthétique et précieuse. [...] Avec la pensée archaïque grecque et surtout avec le monde de l'épopée homérique, nous assistons à une composition subtile qui fait glisser la représentation entre deux domaines: le domaine de la vie quotidienne et de l'expérience visuelle que chacun peut effectuer lorsqu'il observe les rivages de son île et qu'il tient un bouclier de soldat et le domaine de l'imaginaire qui investit plastiquement une image pour la travailler soit sur d'autres objets de la création artistique, soit pour la travailler par la texture des mots et la faire entrer dans le monde du texte afin qu'elle devienne texte du monde ». André (Laury-Nuria), «Entre histoire et géographie, écrire le paysage chez Diodore de Sicile et Strabon. Le cas des îles occidentales ", in Orient \& Méditerranée 19, 2016, à paraître.

23. Nous reprenons en partie quelques uns des développements proposés lors d'une communication donnée en commun avec Sophie Lécole-Solnychkine, "Réflexion générique autour du paysage de l'epos à l'écran : le retour du paysage naturel à l'écran après 2010, le cas de la série Odysseus » lors de la journée d'étude L'epos à l'écran. Interactions génériques entre littérature et cinéma, organisée par Benjamin Thomas et Saulo Neiva , à l'Université de Clermont-Ferrand, 23 novembre 2013.

24. C'est sans doute ce qui a constitué, pour une part, la surprise ou la déception de certains spectateurs. La dimension des realia est donc délaissée, au profit d'une logique de surenchère et 
d'excès qui devient le garant de la dimension auctoriale de l'épos dans ces représentations. Quelle est alors la surprise du spectateur habitué aux péplums grandiloquents, lorsqu'il découvre la série Odysseus : ici, ni décors grandioses, ni reconstitutions paysagères spectaculaires à quelques exceptions près, ni interventions du merveilleux, divin ou surnaturel (dans la mesure où le registre du merveilleux (divin ou surnaturel) offre aux péplums hollywoodiens le prétexte de la spectacularisation des décors et des paysages, et de la débauche d'effets spéciaux qui la rend possible).

25. «[Homère], à vrai dire, ne craint nullement de mêler le sublime et le tragique d'éléments réalistes pris dans la réalité quotidienne; une telle crainte est étrangère à son style et inconciliable avec lui; dans l'épisode de la cicatrice, par exemple, la scène domestique, calmement dépeinte, du lavement des pieds s'incorpore dans la grande action décisive et sublime, du retour d'Ulysse ». Auerbach (Erich), Mimésis. La représentation de la réalité dans la littérature occidentale, Paris, Gallimard, 1977, p. 32.

26. Ce phénomène se laisse appréhender, à l'écran, par des mouvements de resserrement et de rétractation du paysage à l'écran qui donnent lieu à trois types de plans paysagers. Ces trois types sont : une série de plans larges sans personnage et à faible valeur diégétique, des plans plus resserrés qui incluent des personnages en nous les montrant immergés dans le paysage naturel portugais devant signifier le paysage grec d'Ithaque (il s'agit d'une coproduction franco-italoportugaise majoritairement tournée dans le Sud du Portugal pour la beauté de ses paysages et ses rivages singuliers) et enfin, une série de plans larges ouvrant sur le paysage avec à chaque fois des personnages de dos.

27. Ainsi, d'autres épisodes du voyage sont, pour l'un, représenté par une troupe d'acteurs de théâtre ; pour l'autre, raconté par Ulysse lui-même à Orion, mais dans le présent diégétique ; et enfin, pour le dernier, rêvé par Ulysse, en une seule et unique image de flash-back. Cette image, la seule qui filtre du voyage à proprement parler, repousse à nouveau l'épique merveilleux, en nous montrant simplement Ulysse cadré sur un fond de nature, en lieu et place de la rencontre avec Nausicaa. Notons toutefois que cette scène possède également une dimension champêtre dans l'épopée homérique. C'est ce que remarque Graves (Robert), Les mythes grecs, Paris, Fayard, 1967, p. 562.

28. «L'Odyssée est, par excellence, le grand poème du nostos, du retour sans cesse retardé, sans cesse différé à ithaque. C'est pourquoi, chaque fois que je lis ce mot, je ne peux m'empêcher de penser à Ulysse pleurant sur le rivage de l'île de Calypso et suppliant la nymphe de le laisser retourner chez lui. Et chaque fois que l'entends, ce mot, dans un texte ou un poème, je crois entendre aussir sur le sable, l'infini gémissement, ressassement d'une mer dont chaque vague dit en mourant : nostos... nostos... nostos... ", Lacarrière (Jacques), Dictionnaire amoureux de la Grèce ancienne, Paris, Plon, 2001, p. 380-381.

29. Cassin (Barbara), La Nostalgie. Quand donc est-on chez soi ?, Paris, Autrement, 2013.

30. Il lui faut aller: "par les villes, portant dans ses bras une rame polie, jusqu'à ce qu'il parvienne chez des gens qui ne connaissent pas la mer, ni le sel dans les aliments, ni les navires aux joues vermillon, ni les rames qui sont les ailes de navires [...]. Il doit donc repartir vraiment à l'autre bout du monde, au plus loin de l'Odyssée et de la Méditerranée, jusqu'à ceux qui ignorent la mer et la gloire grecque au point de prendre une rame pour une pelle à grain, assimilant ainsi à et par leur culture ce qu'ils ne connaissent pas, "intégrant », dira-t-on sans doute aujourd'hui, l'étrangeté et l'altérité. Je trouve magnifique cette phrase : «quelle est cette pelle à grain sur ta brillante épaule? ", pour dire en toute méprise et en toute douceur le plus lointain du lointain. », Cassin (Barbara), La Nostalgie, op.cit., p. 51-52.

31. Voir le rôle du rivage dans la construction du paysage dans l'épopée, André (Laury-Nuria), De l'île au texte. Le paysage-palimpseste des anciens Grecs, op.cit.

32. Cette rencontre, pour fortuite qu'elle puisse paraître, est en réalité déjà inscrite dans l'univers mental des Anciens qui, à la période impériale (mais peut-être déjà bien avant), ont 
imaginé qu'Ulysse a fondé la ville d'Olisipona, c'est-à-dire l'ancienne Lisbonne. Que ce soit Varron, Pline l'Ancien, ou Strabon, tous ont imaginé que l'infatigable errant odysséen a prolongé l'espace méditerranéen et l'a projeté sur sa façade atlantique, en y inscrivant à jamais son passage par la fondation d'olisipo-Lisbonne. Si l'on rapporte cette question de la fondation odysséenne de Lisbonne, à celle du paysage d'Odysseus, si l'on veut bien élargir l'espace du nostos à celui de la saudade, cette perspective résonne alors favorablement du côté de ce que Marc Ferniot a appelé la topoiétique. Cette attitude ou discipline de l'esthétique, se présentant comme une radicalisation de l'artialisation théorisée par Alain Roger, se propose de singulariser le stéréotype paysager (le lieu, ou le lieu commun, double sens de topos) en faisant jouer les diverses strates culturelles qui constituent l'espace en paysage palimpseste de culture(s). Il s'agirait alors de fictionner figures et lieux communs, par l'activité d'une lecture plastique du paysage, qui mènerait à dialectiser les topoï de notre culture. Par le biais d'une lecture topoï̀te de l'Odyssée, la série Odysseus métisse le nostos et la saudade et c'est ainsi l'espace d'un dialogue qui s'ouvre entre les lieux communs, et permet l'émergence d'une singularisation, qui actualise une réception de l'épos.

33. Il s'agit de la période charnière qui voit l'essoufflement et l'agonie de la République romaine, épuisée par les guerres intestines et l'avènement du Principat d'Auguste.

34. Pour Vivien Bessières, il ne fait aucun doute que cette série relève du péplum. Il l'indique dès l'introduction de son article consacré à la question du genre de la série: «En tant que série de genre historique, elle avait prétention à représenter le réel, Rome participe d'un genre à prétention réaliste : le péplum, ou plutôt l'epic aux États-Unis. Passant d'un art à l'autre, un tel genre modifie-t-il son rapport au réel ? » Bessières (Vivien) « Rome : un péplum enfin réaliste ? ", TV/Series [En ligne], 1 | 2012, mis en ligne le 15 mai 2012, consulté le 03 août 2016. URL : http:// tvseries.revues.org/1066. Nous sommes, pour notre part, quelque peu gênée par l'association automatique entre le genre cinématographique du péplum et la dimension historique comme si le péplum avait toujours eu l'intention de porter à l'écran un discours historique sinon vrai (et donc tiré du monde des spécialistes) du moins vraisemblable. Nous le sommes d'autant plus que le genre du péplum au cinéma actuellement (comprendre entre les années 2000 et 2015) tend de plus en plus vers le merveilleux, le mythologique, le retravaillé c'est-à-dire vers l'écart d'avec les versions historiques, littéraires, archéologiques etc. antiques et "canoniques». Le critère de vérité historique ainsi que la notion de mimèsis ne semble vraiment plus être ceux qui régissent les logiques de création des mises en image de l'Antiquité à l'écran. Voir à ce propos, André (Laury-Nuria), Game of Rome, op.cit., p. 11. Le péplum n'est pas systématiquement synonyme de film historique selon nous.

35. Voir André (Laury-Nuria) et Lécole-Solnychkine (Sophie), « Paysages et topoï dans le péplum grec contemporain : de l'esthétique de la ruine à sa résurrection virtuelle », op.cit.

36. Extrêmement rare, la 3D se fait d'une très grande discrétion dans cette série: on notera seulement en technique d'incrustation soit dans le décor reproduisant le forum, une reproduction virtuelle de la colline du Palatin surmontée de ce qui pourrait être le temple d'Apollon qui surplombe la ville (notons ici qu'en réalité ce temple est anachronique, il date de l'époque impériale et non républicaine, il faut inauguré en 28 av. J.-C., à l'époque républicaine le Palatin est surtout recouvert des maisons patriciennes ; il faudra attendre Tibère pour qu'il y ait véritablement un grand palais impérial sur le Palatin) soit, dans le paysage naturel de la campagne italienne une vue lointaine de la ville de Rome qui se découvre aux héros Titus Pullo et Licius Vorenus, notamment lors de l'avancée des troupes de César vers la ville de Rome aux épisodes 1 et 2 de la saison 1 : cette vue sera reprise autant de fois que nécessaire lors des allers et venues entre les paysages extérieurs, ceux des campagnes militaires et la ville de Rome, centre des intrigues. Notons enfin quelques utilisations, toujours aussi discrètes, de la 3D dans la saison 2 , notamment pour les vues du port d'Alexandrie. 
37. Le travail de reconstitution des décors historiques a nécessité une équipe de plus de 350 personnes.

38. Pour une étude de ce corpus voir les travaux d'Alix Barbet, d'Agnès Rouveret et de JeanMichel Croisille. Au sein de leur immense bibliographie, nous n'avons sélectionné qu'un seul ouvrage de référence : Barbet (Alix), La Peinture murale romaine. Les styles décoratifs, Paris, Picard, 2009 (2e édition), Croisille (Jean-Michel), Paysages dans la peinture romaine, Paris, Picard, 2010 et Rouveret (Agnès) et alii, La Peinture romaine, Actes Sud, Arles, 2006.

39. Dans la mesure où les décors, les peintures, les céramiques et sculptures sont reproduites à l'identique du modèle antique dont ils sont l'ersatz dans la série, on peut considérer qu'ils «valent » pour le modèle antique et pour la question de l'ornement au sens théorique du terme.

40. Nous parlerons, par convention de la géographie et du paysage fictifs à savoir que lorsque nous parlons de Rome, il s'agit de la Rome montré à l'écran, celle de la fiction télévisuelle pour laquelle nous espérons que le lecteur comprendra bien, au vu des informations données précédemment, qu'il s'agit, dans la réalité du plateau de tournage, des décors de Cinecittà. Il en va de même pour la mention des autres paysages naturels, le Latium, les plaines des Gaules, les rivages d'Illyrie, etc. : il s'agit des paysages naturels d'Italie où la production a choisi de tourner en plein air.

41. On citera en partie l'épisode 1 qui montre la reddition de Vercingétorix, l'épisode 07 de la poursuite de Pompée par César et l'épisode 8 montrant les campagnes de César en Égypte et à Alexandrie.

42. Nous commentons ici ce qui semble être la vision des producteurs de la série télévisée, pour une critique de cette approche voir Dupont (Florence), "Rome, ton univers impitoyable ", Le Monde diplomatique, 2007/4, $\mathrm{n}^{\circ} 637$.

43. Bien que fort justement critiqué par le personnage d'Atia qui nous donne à comprendre les jugements de valeurs et de goûts de nobles romains.

44. Le principe analysé ci-dessus se répète dans l'ensemble de la série avec une subtilité qui ne s'essouffle jamais durant les deux saisons.

45. André (Laury-Nuria), Game of Rome, op.cit., p. 54.

46. Voir André (Laury-Nuria), Game of Rome. L'Antiquité vidéoludique, op.cit, p. 77.

47. Voir André (Laury-Nuria), Game of Rome. L'Antiquité vidéoludique, op.cit.

48. Mais qui pourrait reprocher à une série visant un large public avec seulement deux saisons de 12 épisodes de ne pas fournir un travail «juste » scientifiquement et aussi érudit que celui d'un historien pour lequel, en réalité, nous savons bien qu'il faut toute une vie de travail forcené... (ce n'est tout simplement pas le propos d'une série !). Bien que Florence Dupont ait raison (Dupont (Florence), «Rome, ton univers impitoyable... », op.cit.), sur le plan historique et anthropologique de nous mettre en garde contre des "erreurs» de lecture dues à notre vision européenne et moderne, nous ne pouvons disqualifier sur le plan esthétique, le travail inventif et créatif de cette série et de ce générique en particulier. Il emblématise à nos yeux un processus de réceptioncréation de la matière antique qui la transforme en espace de partage. 


\section{AUTEUR}

\section{LAURY-NURIA ANDRÉ}

Docteur de l'ENS de Lyon, Laury-Nuria André enseigne dans le Supérieur les Langues anciennes et l'Histoire de l'Art antique. Spécialiste de l'épopée et du paysage, plasticienne et romancière, elle interroge la place de l'Antiquité dans la culture contemporaine. 